\title{
On solutions of fundamental problems of physics and the contours of new physics
}

\begin{abstract}
Zahid Zakir
Abstract

In quantum and gravitational physics, a large set of fundamental problems have accumulated over the past hundred years, which shows the incompleteness of the formation of basic theories. In the paper solutions a number of such problems, presented by the author recently, is reviewed. Unlike other hypothesis-based attempts, the new solutions are based on physical principles and empirical facts, which makes the new formulations of basic theories more consistent. Problems of a "technical" nature, arising due to deviations from known principles, are solved by consistently following them. Other problems were solved by introducing new principles or changing old ones, but also on the basis of facts. Consequently, these solutions in this or a more refined form will enter the new physics initiated by them. The contours and prospects of this new physics are considered. The reasons of the long time crisis in fundamental physics and ways to overcome it are discussed. It is shown that the main reason for the crisis were deviations from the methodology and ethics of natural sciences, and the new physics began to form as a result of the return to them.
\end{abstract}

Keywords: diffusion quantum mechanics, diffusion gravity, quantization of fields, microcausality, vacuum energy, gravitational regularization, renormalization, quantum gravity, gravitational collapse, frozars, cosmological redshift, slowing time cosmology, local and global relativity, methodology, scientific ethics.

\section{Content}

Introduction

1. On the crisis in fundamental physics and the ways to new physics ................................3

1.1. The crisis in the foundations of modern physics and its origins. ...................................... 4

1.2. What were the deviations from the methodology and ethics of natural sciences? ..............5

1.3. Returning to the methodology and ethics of natural sciences as a way to new physics..... 7

2. Fundamental problems of quantum mechanics and their solutions .............................11

2.1. What are the physical principles of quantum mechanics and what was the need for them? 12

2.2. The problem of the source of quantum fluctuations and the Munchausen paradox..........13

2.3. Why in quantum theory the amplitudes of probabilities are summed? ............................. 14

2.4. Are there hidden parameters and what is the reason for nonlocality? ............................ 14

2.5. What is the physical meaning of the uncertainty relations and quantum potential?........ 15

2.6. What is the physical meaning of the light velocity and its constancy?........................... 15

2.7. What is the physical meaning of the rest energy of particles? ....................................... 16

2.8. How quantum statistics and particle indistinguishability arise? .................................... 16

3. Problems of quantum field theory and their solutions...................................................18

3.1. How to quantize the negative energy states correctly? ................................................ 19

3.2. Are there zero-point fluctuations of vacuum fields?........................................................ 20

3.3. How to quantize strings and do they have critical dimensions? ......................................20

3.4. What are the consistent conditions of microcausality? ................................................... 21

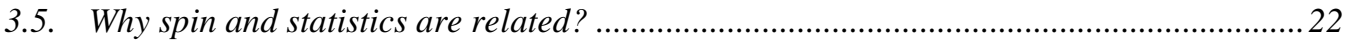

3.6. Are high-order perturbation theory corrections infinite? ............................................. 22

3.7. Are chiral anomalies infinite and is their cancellation in SM accidental? ......................23

\footnotetext{
${ }^{1}$ Center for Theoretical Physics and Astrophysics, Tashkent Uzbekistan, zzakir@ qgph.org, ORCID
} 


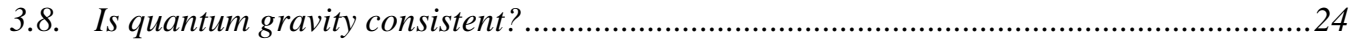

3.9. How to describe the possible substructure of fundamental particles? .............................24

3.10. Is there a need for supersymmetry?..........................................................................25

4. Problems of gravitational collapse and their solutions...................................................26

4.1. How to describe structure and evolution of an extended object in GR?..........................26

4.2. Can the surface of a collapsing star cross its gravitational radius and fall to the center? 27

4.3. How does the shell collapse and will it have a horizon and a singularity?......................28

4.4. How a dust star collapses and how its structure evolves? .............................................29

4.5. How does a rotating star collapse and will it have an ergosphere? ................................31

4.6. When a relativistic explosion of a collapsing star can occur? ...........................................32

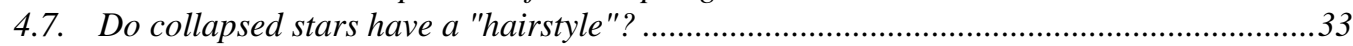

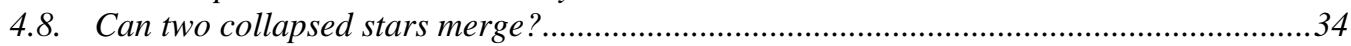

4.9. How supermassive collapsars differ from ordinary ones? ...............................................35

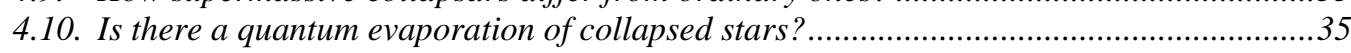

5. Problems of the theory of gravity and their solutions ...................................................36

5.1. Was Maxwell right about the nature of gravity? ........................................................... 36

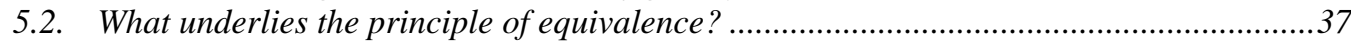

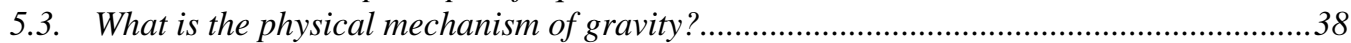

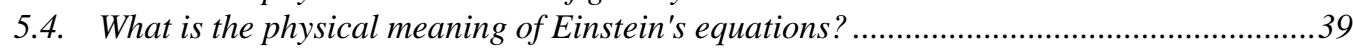

6. Problems of cosmology and their solutions ...................................................................39

6.1. The double redshift paradox in relativistic cosmology..................................................40

6.2. Is it necessary to take into account aberration in cosmology?........................................41

6.3. Does the rate of proper times change during expansion? ................................................41

6.4. Are there cosmological constant and dark energy? ......................................................42

6.5. Problems of Friedmann models of cosmology and their solutions..................................43

6.6. Does dark matter exist?..............................................................................................43

6.7. What are the curvature and global structure of the universe? ........................................44

7. From SR and GR to local and global theories of relativity .........................................44

7.1. LR is a local form of $S R$ and for this reason it is universal..........................................45

7.2. $G I R$ as a physical part of GR on hypersurfaces of simultaneity......................................46

7.3. Consequences of LR and GlR for quantum and gravitational physics ............................48

8. The contours and perspectives of new physics..........................................................49

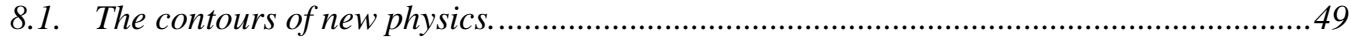

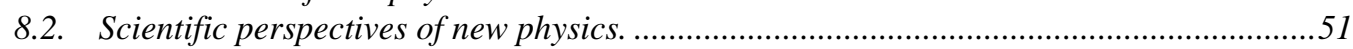

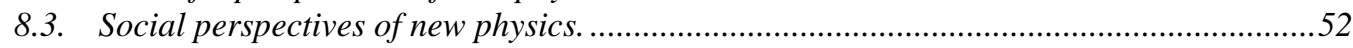





\section{Introduction}

Relativity theory and quantum theory, together with their applications to particle physics, astrophysics, and cosmology, form the theoretical foundation of modern physics. They describe most of the known facts, predicted many new ones and have been repeatedly confirmed. Nevertheless, many fundamental unsolved problems remained in the foundations of these basic theories and in their important applications. Moreover, due to ambiguities in the definition of basic concepts and physical principles, they still could not become consistent physical theories, i.e. besides particular problems, there was also a common problem - their incompleteness.

The list of unsolved problems in physics is wide enough and is often given in the literature. But only some of them are fundamental, i.e. relate to the principles of physics or their most important consequences. Moreover, many unsolved problems were classified as "resolved" only because of the existing consensus on the "correctness" of one of the hypotheses. The solution to at least some of these fundamental problems will remarkably change the physical picture of the world [30,35]. 
The present paper provides an overview of those fundamental problems for which the author has proposed new solutions presented in recent papers in 2020 [39-44] and which are systematically presented in the books [45-49] (earlier publications of the author contained only trial versions). Unlike other authors, who proceeded from hypotheses without an experimental basis, the developed approach proceeds from physical principles and facts. This way of solving problems of physics, as the history shows, is the most reliable and its results are trustworthy. It is usually quite productive too, since giving explanations for previously inexplicable phenomena, it also leads to unexpected predictions.

The easiest to understand are solutions to fundamental problems of a "technical" nature, i.e. problems arising due to inadequate application of the principles of basic theories or deviations from them. They are solved by consistently following these principles, correcting distortions and errors in the old paradigm that arose because of these problems. These solutions thus complete the formation of basic theories, which were delayed due to these deviations.

More radical are solutions related by the introduction of new or change in known principles. But even in these cases, they are not hypotheses, but the conclusions from known facts. In physics as natural science, any change in paradigm associated with the formulation of new or clarification of known principles opens up new perspectives and, therefore, begins a new stage of development.

In the Part 1 of the paper the reasons for the almost half-century crisis in the foundations of physics and the ways to overcome it are discussed. It is shown that the main cause of the crisis was a departure from the methodology and ethics of natural sciences, and the new physics is the result of a return to them.

Solutions to particular problems are grouped into six parts (Parts 2-7), each of which is independent, although the results of all parts are mutually consistent. Therefore, each of the parts can be read independently, starting with those that are more interesting to the reader. In the last part (Part 8), the contours and perspectives of the new physics, following from the proposed solutions to the problems, are considered.

List of abbreviations: SR - special relativity, GR - general relativity, QFT - quantum field theory, SF - Stueckelberg-Feynman, SM - Standard Model, DQM - diffusion quantum mechanics, STC - slowing time cosmology, FR - frame of reference, LFR - local FR, GFR - global FR, LR - local relativity, GIR - global relativity.

\section{On the crisis in fundamental physics and the ways to new physics}

In the last almost half a century, after the triumphant discovery of neutron stars in the late $60 \mathrm{~s}$, and then the completion of SM of particle physics in the mid-70s, fundamental physics was in a deep crisis. Everything that was discovered at that time in experiments was predicted before 1975 in the framework of GR, QM, QFT, and SM. However, a number of fundamental problems remained unresolved both in these theories and in the description of phenomena beyond them. But the theoretical models created to solve these problems have not been confirmed, and moreover, they created more problems than solved [30]. The reasons for this long time crisis and ways to overcome it are discussed below.

1. The crisis in the foundations of modern physics and its origins.

2. What were the deviations from the methodology and ethics of natural sciences?

3. The way to new physics is in a return to the methodology and ethics of natural sciences.

The problems of the methodology of physics will be discussed in more detail in the book [49]. 


\subsection{The crisis in the foundations of modern physics and its origins.}

The set of fundamental problems of physics, which will be considered in this paper, arose mainly in the first half of the 20th century and took its final form in the 1970s, and since then this set has remained practically unchanged. It became an organic part of the current paradigm, expressing its internal contradictions, as well as contradictions with a number of known facts.

As the reasons for such a long stagnation, in fact, a crisis, the lack of experimental facts necessary for further progress and the impossibility of testing the leading hypotheses in the foreseeable future were indicated [30,35]. However, this paper shows the opposite, that such facts were enough for normal development and thus this does not explain such long crisis.

If the crisis was not inevitable, then it could have occurred due to a lack of resources human and otherwise - compared to the scale of the tasks, as well as external reasons unfavorable for the scientific community. But, by the irony of history, in the last half century, fundamental physics had more resources, human and otherwise, and external conditions favorable for development than in its entire history before that. Therefore, everything happened just the opposite, and the longest crisis in the history of physics occurred precisely when there were all the conditions for rapid development.

How could such a crisis occur in fundamental physics in the absence of internal and external objective reasons? Naturally, only due to subjective reasons, crisis processes within the scientific community itself.

The first of these reasons for the crisis, as will be shown in the next Section, was a deviation from the methodology of natural sciences in solving fundamental problems of physics and the promoting the resulting untenable paradigm changes by extra-scientific methods. This led, firstly, to the inclusion into the paradigm in some cases of two incompatible explanations of a physical phenomenon as compatible ones, and, secondly, to the replacement of the analysis of facts with mathematical hypotheses with non-physical entities contradicting to these facts. All this divided the scientific community into two incompatible parts - supporters and opponents of such distortions of scientific methodology. At the same time, each of these parts of the community began to degrade - the first because of the achieved de jure monopoly on truth, the second because of being pushed aside to the position of dissidents only because of the fidelity of scientific methodology, and this became the catalyst for a systemic crisis.

The first reason led to two more other reasons that deepened the crisis, turning a purely scientific crisis into a crisis of the entire scientific community. These reasons were systematic distortions of scientific ethics and science policy. Naturally, the departure from the methodology of natural sciences led to objections and alternatives. But the majority of the scientific community, united around the standard paradigm and leading hypotheses, in the absence of logical answers to opponents, not only began to ignore criticism, but also to supersede the supporters of reasonable alternatives from the scientific literature and the scientific community. A distortion of scientific ethics and science policy was also the imposition of unconvinced doctrines on the scientific community, the education system and the general public by methods of large-scale propaganda. As a result, both the scientific community and the organization of science as a whole get on the way of conservation of the standard paradigm and leading search directions, which led to stagnation instead of development. Notice, without delving into the problems of the scientific community, that a society, supporting fundamental science as one of the basic values of civilization, was not ready for such a situation and did not have mechanisms to assist fundamental science to overcome such a long time systemic crisis.

The uniqueness of this crisis lies in the fact that if before that every epoch in the history of physics gave confirmed solutions to a large number of fundamental problems, then after such a "putsch" in the methodology and organization of science, fundamental physics turned out to be practically fruitless, and reached a dead end. The huge resources of society, both human and otherwise, turned out to be directed mainly towards illusory goals, towards "fight windmills", which is ordinary for mathematical-minded people, but unnatural for physicists. 


\subsection{What were the deviations from the methodology and ethics of natural sciences?}

Deviations from the methodology of natural sciences, as involuntary and temporary ones, were already admitted in basic theories, in quantum mechanics, QFT and GR, and during the crisis they, instead of liquidation, were brought to the point of absurdity. These deviations consisted in reconciling with contradictions, practicing so-called doublethink, instead of actually resolving them, as well as inflating the non-physical parts of basic theories by describing illusory worlds, instead of being limited to their physical parts and describing reality.

\section{A. Doublethink.}

In a number of cases, two opposite explanations of the same physical phenomenon were accepted as equally admissible. This is known in psychology as the phenomenon of "doublethink", the ability of people to hold two opposite beliefs at the same time. At doublethink, the phenomenon in one case is explained by the desired unreal reason, without taking into account the real reason, and then, when it is necessary to explain also through the real reason, the unreal one is not taken into account. As a result, a false belief is created that unreal and real reasons are equally acceptable.

Doublethink is characteristic for communities where like-mindedness is established not only in what is true, but also in what has not been proven to be true, but it is beneficial to the dominating part of the community. In a scientific community, where the supporters of doublethink dominate, instead of using scientific methodology to resolve contradictions, reconciliation with them is required, and like-mindedness and agreement on this issue become the conditions for belonging to the dominant part or to the scientific community in general.

In Section 1.3 the cases of doublethink in quantum and gravitational physics are presented. In quantum physics these are the cases of the wave-particle duality, Munchausen's paradox, the zero-point vacuum fluctuations and strong microcausality. In gravitational physics, these are the treatments of gravitational redshift, gravitational time dilation, gravitational collapse, redshift and aberration in cosmology. In each of these cases, it is also shown how a return to the methodology of natural sciences excludes previous doublethink.

In particular, wave-particle duality consists in the fact that in an ensemble of experiments carried out each time with one electron, this ensemble demonstrates wave properties, but when the wave properties were attributed to each electron, it was doublethink. Based on this, absurd statements were made that an electron is capable of simultaneously passing through two, three and hundreds slits on a screen in its way, and then on the second screen to interfere with itself. The initial assumption that fluctuating microparticles are isolated and capable of spontaneously changing their energy and momentum led to this absurdity. As will be shown in the paper, when the question about the source of fluctuations of microparticles is raised from the very beginning and the correct answer is given, then the rest of the questions are solved without any absurdity.

Another example of doublethink is the zero-point fluctuations in the vacuum of quantum fields. The well-known effects, the Lamb shift and the Casimir effect, are completely explained by the electromagnetic fields of real sources. But, if one can switch off (!) these real sources and assumes the existence of the zero-point vacuum fluctuations, then these two effects can also be fully explained. On this basis, in the standard paradigm, it was accepted to consider the reality of the zero-point vacuum fluctuations as "proven". However, the question of how experimenters could in the real world "turn off" the influence of the real sources was not specified in any way, which is typical for other cases of doublethink also.

Doublethink in the case of the zero-point fluctuation has led, in particular, to string models. All the "business" in these models was based on the hypothesis about the zero-point fluctuations. Since this led to some anomalies, then to eliminate them, the dimension of space had to be equal to the critical one, which fixed the symmetries and properties of particles and fields. But, as it turned out, fields and strings can and should be quantized without zero-point fluctuations, which makes string models trivial due to the lack of critical dimensions. 
These and other examples of doublethink are discussed in Section 1.3, and they are all based on the same departure from the methodology of natural sciences, while a return to it and the search for real solutions to problems excludes doublethink.

\section{B. Plunging into the non-physical parts of basic theories and fictitious worlds}

The basic theories, on the one hand, contained a physical part, on which all their successes and authority were based, and on the other hand, their historical formulations also included a non-physical part, introduced by the founders temporarily in the hope that new generations would be able to exclude it from theory.

However, instead of this step required by the methodology of natural sciences, next generations of researchers began to do exactly the opposite. Instead of limiting the physical parts of basic theories, they began to develop their non-physical parts. The non-physical parts were considered legalized due to they were in the basic theories and it was ignored the fact that they, unlike the physical parts, are not confirmed.

An example is the above mentioned situation in quantum theory. In radioactive decay, it was assumed that a particle supposedly leaves the potential well without external help. Here, the choice of the particle itself as the source of the energy it needs for such an exit means attributing to it the "Munchausen's" ability to pull itself out of the swamp (see Section 1.3 about the "Munchausen paradox"). In quantum mechanics, free particles were considered as isolated ones, as in classical mechanics, but with "quantum" properties, i.e. able to accidentally change own energy and momentum. But it violated one of the basic laws of physics for an isolated object - the energy and momentum conservation in a homogeneous and isotropic space.

Even stranger and unacceptable from the point of view of the methodology of natural sciences was the ignoring in the standard formulation of QFT the effects of gravity in loop diagrams, where the physical energy of quanta in intermediate states can be very large. Based on this temporarily introduced simplification that becomes illegal around the Planck energies, it was further concluded that the contributions of such diagrams to the characteristics of particles (masses, charges, process probabilities) are "infinite." To eliminate these fictitious "infinities", large-scale research programs were then developed over many decades.

And, finally, the formalism of GR allowed the introduction of arbitrary frames of reference, although, according to the equivalence principle, only those related by local Lorentz transformations were locally admissible, and all the others were nonphysical. In GR, a cosmological constant of any sign was also allowed. On the formal "admissibility" of the nonphysical frames of reference in GR, the hypotheses about black holes and unremovable "infinities" in quantum gravity are based. On such "admissibility" of antigravity with a constant energy density in the expanding space is based the SM in cosmology.

The most dramatic was the departure from the methodology of natural sciences in the theory of stellar collapse. This theory reached its highest point in the 1939 Oppenheimer-Snyder paper [23], where a set of simultaneously occurring events along the world lines of particles inside a homogeneous dust star was found. It was the physical solution to the collapse problem with the correct taking into account the initial and boundary conditions, and according to it the collapsing star quickly freezes on the hypersurfaces of simultaneity. However, the scientific community did not understand the Oppenheimer-Snyder solution and follow a false scent of ignoring it. This ignoring was done by replacing it with the local Friedman solution, which coincides with the Newtonian one (presenting Friedman's solution as the "Oppenheimer-Snyder solution"). The result was the Newtonian theory scenario of the collapse with the formation of "black holes". The crossing of gravitational radius by the stellar surface, which is declared by this scenario, in GR cannot occur in a finite time of the external world and therefore is a nonphysical "event". The true Oppenheimer-Snyder solution, describing physical events during the collapse of entire star, was restored in the frozar theory (see Part 4).

In this regard, it should be recalled that the methodology of mathematical physics, whose priority was one of the main reasons for the crisis, is principally different from the methodology of theoretical physics. In theoretical physics, a mathematical model is true only when it 
describes reality and this is confirmed experimentally. In mathematical physics, however, the study of models of worlds more interesting and diverse than the real world is a normal scientific activity, and the concept of truth is narrowed down to the level of internal consistency of a mathematical model. The richness and beauty of these worlds often lured physicists, while the mission of the latter is to study only our reality as it is. Many founders of physics fell into this trap of mathematical power and beauty, but before this did not lead to such a long dominance in the current paradigm of fundamental physics of the fictitious worlds of mathematical physics.

During the crisis, these deviations from the methodology of natural sciences became the norm and they were legalized for new hypotheses also. Moreover, the latter purposefully reinforced and systematically developed precisely these deviations. At the same time, the elimination of the physical restrictions in the construction of worlds more interesting than reality became the strongest doping and the scientific community plunged into these wonderful worlds for a long time and practically forgetting real physics.

Thus, reconciliation with doublethink and legitimization of the non-physical parts of the basic theories of physics by appealing to their experimentally tested physical parts, as well as the unreasonable mathematization of theoretical physics, led to a departure from the methodology of natural sciences, and further steps on this doubtful, and often simply wrong way were predestined to failure initially. As a result, practically none of the fundamental problems of physics was solved, even those of them for the solution of which there were already all the conditions. A return to the methodology of natural sciences, as will be shown below, allows one to find reasonable solutions for most of these problems without new hypotheses and opens the way to new physics.

\subsection{Returning to the methodology and ethics of natural sciences as a way to new physics.}

The methodology of physics as a natural science is based on the accumulation and analysis of experimental facts and their explanation by putting forward hypotheses and constructing theories on their basis. Fundamental physical theory proceeds from physical principles based on experiments and allows one to describe and explain by deduction all other facts in the field of its applicability.

A hypothesis contains at least one initial assumption, or a mathematical axiom, which does not directly follow from experiments. If one of these hypotheses describes most of the facts, or even all known, then it becomes a candidate for a working theory. If the predictions of the hypothesis are confirmed, i.e. it allows one to discover new facts, then it becomes a confirmed working theory. Nevertheless, until the initial postulates of this working theory are not directly or indirectly confirmed by experiments, it remains only a reliable mathematical model only allowing one to describe the phenomena, but does not explain them.

\section{A. Exclusion of non-physical simplifications and generalizations in basic theories}

The hypothetical postulates of the working theory are often formulated in direct analogy with the previous outdated theory, assuming the same simplifications and idealizations. This extrapolation is sometimes confirmed and sometimes not. If not, then it is necessary to clarify the postulates of the working theory, taking into account new conditions.

Sometimes the postulates of the working theory are formulated more general than they are in the real world. Such a theory will have both physical and non-physical parts and confirmation of its physical part does not concern its non-physical part. Therefore, it is necessary to separate these two parts and exclude from the theory its non-physical part.

For historical reasons, in the formulation of the initial postulates of the basic theories of physics, both of these above mentioned cases, unreasonable simplifications and excessive generalizations, took place, and therefore appropriate clarifications were required. This is one of the conditions for the transition of the basic theories of physics from the stage of a working 
theory to the stage of a consistent physical theory describing the real world only. Let's briefly consider the main of these refinements.

Quantum mechanics in the form of its formalism based on purely mathematical axioms has also remained a working theory until now. The formulation of those physical principles from which the formalism of quantum mechanics follows will turn this working theory into a complete physical theory. We can be sure that this theory will not contain the above (most likely nonphysical) hypothesis that the energy and momentum of an isolated particle may not be conserved. The desire to keep the surrounding background empty could not be a reason for abandoning such a basic law of physics as the conservation of energy and momentum of an isolated object. Therefore, Einstein was right, who formulated it metaphorically as "God does not play dice." In the DQM (see Part 2), the Munchausen paradox is absent, since the particles are classical, and the source of their fluctuations is the action of the background field on them. When, under the influence of fluctuations of this background, the energy of the particle increases for a short time, the energy of the background field in this place will decrease by the same amount, which leads to the conservation of the energy of the entire system.

A number of artificial procedures were also introduced in QFT, both at quantization of fields and at taking into account or ignoring known phenomena and facts. A more accurate formulation of the initial physical principles of QFT should make it possible to get rid of the indicated procedures and simplifications, as well as from their absurd consequences in the form of infinities.

GR, based on the mathematical principle of general covariance, also remained a working theory, since this principle, being too broad, was not adequate to the properties of physical coordinates. But if the formalism of GR is limited, according to the principle of equivalence, to the local Lorentz invariance, and the frames of reference are built from standard scales and standard clocks with their synchronization (when it is possible), then it becomes a consistent physical theory that proceeds from physical principles and operates with physical coordinates.

In the methodology of natural sciences, if a phenomenon is assumed to have two simultaneously influencing reasons, then the consequences of their influence are also taken into account simultaneously. If the phenomenon is explained by only one of the indisputable reasons, then the second reason is considered absent and rejected.

If all this is done according to the methodology of natural sciences, i.e. by searching for physical principles and following them, avoiding contradictions, the new physics that will arise will not only describe the phenomena and structures of physics, but also explain them, and also open up new perspectives. In the paper the most probable, in the opinion of the author, solutions to a number of fundamental problems of physics, which were found precisely as a result of following this methodology, are presented.

Below the cases of doublethink in quantum and gravitational physics are presented. It is also shown that they are excluded at returning to the methodology of natural sciences.

\section{B. Eliminating the cases of doublethink in quantum theory}

1. Wave-particle duality. The presence of wave properties of an ensemble of particles underlies quantum mechanics. However, the attribution of wave properties to each of the particles in this ensemble, generally accepted as the standard treatment, is an example of doublethink. The assertion that in the experiment with two slits an electron, practically a pointlike particle, allegedly passes through both slits simultaneously and therefore interferes with itself, is absurd. The impossibility of explaining the wave properties of an ensemble of particles appeared due to the extrapolation of the concept of an isolated particle in classical mechanics to microparticles. Part 2 it will be shown that microparticles cannot be considered as isolated ones, since they fluctuate and must have an external source of fluctuations. But if there is such a source, a background field, then the microparticles can be considered as classical ones, and their ensemble as performing conservative diffusion in this field. In the experiment with slits, an electron passes only through one of the slits, but the background field "knows" whether the other slit is open 
or closed. Therefore, with multiple repetitions, i.e. in an ensemble of experiments, the conservatism of diffusion leads to wave properties.

2. The Munchausen paradox. In empty Euclidean space, the energy and momentum of an isolated object are conserved and all of classical physics is based on this. In quantum mechanics, space is the same, but micro-objects supposedly have "quantum" properties, i.e. able to fluctuate spontaneously changing their energy and momentum. In a homogeneous and isotropic space, a "quantum" particle is assigned properties that are forbidden in such a space, and this is doublethink. Einstein objected to this, regarding it as a sign of the incompleteness of quantum mechanics. At fluctuating with sufficiently high energy, a "quantum" particle has a finite probability of leaving the potential well, overcoming a barrier of a finite height and width. The paradox is that such processes are actually observed, although the particle has nowhere to take such energy. The uncertainty relation does not explain this, since it itself is a consequence of fluctuations. The situation is the same as in the well-known myth that Munchausen pulls himself and the horse he is sitting on out of a swamp by his own hair. The choice of the particle that emerged from the potential well as the source of the energy it needs is not a fact, but the same myth about the miraculous (Munchausen's) ability. In DQM, the particles are classical and fluctuate in the background field, and there is no Munchausen paradox here. When the energy of the particle increases at fluctuations of the background field, the energy of the background field in this place decreases by the same amount, which ensures the conservation of the total energy of the system as a whole.

3. The zero-point vacuum fluctuations. A number of well-known effects of particle physics (the Casimir effect, Lamb shift, etc.) can be fully explained only by the zero-point fluctuations of the electromagnetic field vacuum, under the artificial assumption that other sources do not contribute to these effects. On this basis, the myth was created that these experiments confirmed the existence of the zero-point fluctuations and the zeropoint vacuum energy, and this treatment was widely promoted. But the same experiments are fully explained by the fields of real sources (fields of crystal atoms, loop corrections), under the assumption that the zero-point fluctuations are absent. To admit the coexistence of two mutually excluding each other explanations of the same experiments is doublethink. In Part 3 it is shown that there are no zero-point fluctuations in QFT and only the second explanation is correct. The very first "explanation" on the basis of the zero-point fluctuations became possible only because the contribution of real sources was illegally "put out of action", which was the deviation from the methodology of natural sciences. If, nevertheless, insist on the contribution of the zero-point fluctuations, then the two types of contributions should have been taken into account together, and then the predicted values of the effects would be twice as large as those observed.

4. Microcausality. Until now, it was supposed that the microcausality condition of the classical theory of relativistic fields remains valid in QFT and the measurement of the field at one space-time point (event) does not affect the measurement at another point if these points cannot be connected by a light signal This was expressed in the requirement that two operators of fields from these points (anti)commute. At the same time, the causal propagator for field quanta did not disappear behind the light cone, and therefore, superluminal displacements of quanta effectively took place in QFT, albeit on the scale of their Compton length. If the propagator allows superluminal signals (albeit in a mild form, i.e., in a small neighborhood), then the classical causality based on the complete absence of such signals is no longer valid and the measurement at one point can affect the measurement at another point even outside the light cone through virtual quanta carried by the causal propagator. In relation to microcausality in QFT, thus, there was doublethink. In Part 3, it is shown that at quantization of fields in the SF treatment, the field operators at two points do not commute behind the light cone and behave similarly 
to causal propagators. This leads to a soft form of microcausality in QFT, eliminating doublethink in this matter.

\section{Eliminating doublethink in gravitational physics}

1. Gravitational redshift. Photons emitted near a massive body, at detecting by a more distant observer, contain a redshift. In Newtonian theory, this was explained by the fact that photons were emitted with the same frequency as far from the body, but as they escape the body, their energy, and hence frequency, gradually decreased due to work against the force of gravity. In GR, this is explained in the opposite way - the photon's frequency in terms of the distant observer time was redshifted already at emission, since here the local proper time is dilated, and on the way this frequency and corresponding energy of photon remain unchanged. These two explanations of redshift, Newtonian and Einstein's, mutually exclude each other, but they have been treated as almost equivalent ones, which was the first case of doublethink in the treatment of gravitational redshift. Moreover, although the value of the redshift in the first approximation is the same in both theories, the measurement of this effect in experiments was considered to be a confirmation of GR, and the effect itself has long been presented as one of the three "classical" effects of GR. This was the second doublethink in the treatment of gravitational redshift. Later, this second doublethink, attributing the redshift effect to GR, was rejected. The fact that the gravitational redshift is associated with the dilation of the rate of proper times at strong gravity and therefore the Newtonian treatment is erroneous was proved experimentally in the 1970s by comparisons of the clock rate in two places - near and far from the body. But, nevertheless, the first doublethink about the equivalence of the two treatments has not been eliminated.

2. Gravitational time dilation. In flat space-time there are two types of relativistic dilation of proper times - between inertial FR, which is relative, and in non-inertial FR, which is absolutely and well known from the clock (or twin) paradox. In accordance with the principle of equivalence, time dilation in a gravitational field is the same as non-inertial time dilation, i.e. also absolute. For a distant observer, processes near a massive body are slower than for him. For an observer near the body, on the other hand, processes in the distance are faster than for him. Thus, both observers say the same thing, only using their time unit as a standard one. In the standard paradigm, on the one hand, the property of the absoluteness of gravitational time dilation is recognized (red or violetshift when photons rise or fall), but on the other hand, in a number of applications it is illegally substituted by the property of relativity of inertial time dilation, then drawing conclusions based on this "relativity time". The treatment of gravitational time dilation as absolute in one case and relative in the other is doublethink. In Parts 4-5 it is shown that the gravitational time dilation, like the time dilation in the clock paradox, is irreversible and absolute, and this fact eliminates doublethink in its treatment.

3. Gravitational collapse. As a result of the above-mentioned substitution of the absolute gravitational time dilation by the relative time dilation inherent only in inertial FR, a "standard" description of the collapse of stars with the formation of a black hole arose. It was argued that, on the one hand, for a distant observer, a collapsing star turns into a frozen star with a surface that never crosses gravitational radius, and on the other hand, for an observer on a star, the same surface easily and quickly crosses this radius. This doublethink in the fate of a star is a consequence of the previous doublethink in the treatment of gravitational time dilation. In reality, both descriptions give the same picture when taking into account the absoluteness of the dilation of proper times w.r.t. the world time of a distant observer. The collapse then leads to an absolute slowdown and rapid freezing of the surface and other layers of the star in terms of the world time to form a frozar, an object whose structure is frozen due to its own strong gravity. This is clear 
from the plots of the world lines of particles in layers in the Oppenheimer-Snyder solution of Einstein's equations.

4. Cosmological redshift. Of the two mechanisms of this kind of redshift, the Doppler effect and the stretching of wavelengths during expansion, each of them almost completely explains the observed redshifts. Therefore, if they were taken into account together, the predictions would be twice the observed values. Under such contradictory circumstances, some authors interpreted the observations through the Doppler effect, others through stretching, and then a consensus was established that the second explanation is correct, and the first is only a more visual description of the result of the stretching. In reality, these two mechanisms are independent, since the Doppler effect shifts frequencies at the beginning, when photons are emitted, and the second during their propagation, and therefore both contribute to the observed shift (the double redshift paradox see in Part 6). In any case, at considering one mechanism, the other mechanism was completely ignored, which was a case of doublethink. In Part 6 it is shown that when photons are emitted at earlier epochs, their frequencies do not have to be the same as we have here now. The double redshift paradox is solved in the time dilating cosmology resulting from the DQM. In it, photons are emitted with a violetshift, it is compensated in the way by the redshift and only the result of the Doppler effect is observed.

5. Aberration in cosmology. Aberration is a change in direction towards a light source and its apparent luminosity due to relative speed. In relativistic kinematics, the presence of the Doppler effect also leads to an aberration of the light flux. But in the models of relativistic cosmology, aberration was not taken into account even in those cases when the displacements were interpreted through the Doppler effect, since this would violate the agreement between theory and observations. This was doublethink. Aberration leads to the fact that the receding sources will seem dimmer than those that were at rest in relation to us at the same distance. Ignoring the aberration led to an overestimation of the distances to the sources, or to an underestimation of their absolute luminosities. The dimming then had to be explained by various hypotheses, such as about accelerated expansion, for which another hypothesis, about dark energy, was then introduced.

In the subsequent parts of the paper, solutions to particular problems are presented, which thus turn out to be steps towards a new physics which is initiated by them.

\section{Fundamental problems of quantum mechanics and their solutions}

Quantum mechanics has always been perceived only as a very successful and reliably working mathematical model. The incompleteness of its formation was expressed in the fact that the underlying physical principles, that would follow from the experimental facts, were unknown, and there was also no clarity in its physical interpretation. Therefore, the main problems of quantum mechanics were:

4. What are the physical principles of quantum mechanics and what was the need for them?

5. The problem of the source of quantum fluctuations and the Munchausen paradox.

6. Why in quantum theory the amplitudes of probabilities are summed?

7. Are there hidden parameters and what is the reason for nonlocality?

8. What is the physical meaning of the uncertainty relations and quantum potential?

The solution of the first problem, naturally, refers to radical changes, since new basic principles are being formulated. The proposed solution will be realistic if it is based on the results of experiments. Further conclusions from these physical principles and their direct consequences will make it possible to solve or clarify solutions for the following fundamental problems: 


\section{What is the physical meaning of the light velocity and its constancy? \\ 10. What is the physical meaning of the rest energy of particles? \\ 11. How quantum statistics and particle indistinguishability arise?}

All this will also provide new solutions to key problems in the theory of gravity and cosmology, which are presented in Parts 5-6. These results are contained in the papers [41-42] and in the book [45]

\subsection{What are the physical principles of quantum mechanics and what was the need for them?}

Quantum mechanics underlies modern physics, but until now there was no clarity with its physical treatment, and its formalism was still based on mathematical axioms, and not on physical principles following from experiments. Therefore, replacing mathematical axioms with physical principles would clarify the physical treatment of quantum mechanics and complete its formation as a genuine physical theory.

The first step on this way is to find a physical mechanism that leads to its formalism. So many unsuccessful hypothetical mechanisms have been proposed for this that any new one has only aroused skepticism. This happens when the problem is either unsolvable, or the solution has already been partially found, but it turned out to be spread in different treatments. The first case is unprovable, while the second is quite probable and deserves investigation. In the papers [41-42] the second possibility was considered and some of the previous mechanisms were studied as mutually complementary.

The first guesses about the physical mechanism of quantum mechanics appeared in 1926 when Madelung rewrote the Schrödinger equation in hydrodynamic form [19]. In this form, the difference from classical physics was reduced to the appearance in the Hamilton-Jacobi equation of a quantum potential generated by a certain medium.

The idea that quantum mechanics is a theory of the motion of classical particles in a certain medium was supported by two observational facts. First, the particles always fluctuated, and the waves associated with them turned out to be probability waves. Second, localized states of an ensemble of free particles spread out over time. These facts and the form of the wave equation led Ehrenfest [2], Schrödinger [28] and Fürst [11] to an analogy with a new kind of diffusion.

This specific diffusion had to be frictionless, i.e. conservative. For this reason, in stochastic treatments [6,15], attempts were made to obtain conservative diffusion from two families of Brownian motions. But later it was shown that quantum mechanics cannot be reduced to such simple Markov processes [12]. Nevertheless, this attempt gave something more valuable - the formalism of conservative diffusion, which turned out to be little dependent on the particular mechanism of fluctuations [13].

In addition, the analogy with diffusion in its integral representation formed the basis of the physically most descriptive of the standard quantization methods - Feynman path integrals [7], a complex version of Wiener path integrals in the case of ordinary dissipative diffusion.

In the first of the papers [41] the required physical principles of quantum mechanics were formulated. The observational fact that a wave packet describing a localized ensemble of microobjects spreads out according to the diffusion law implies the quantum principle of equivalence, according to which the motion of an ensemble of quantum objects in empty space is equivalent to conservative diffusion of an ensemble of classical objects in a fluctuating background field.

It was also shown that observations confirm the validity of the principle of relativity for quantum phenomena, formulated as the principle of constancy of quantum fluctuations, namely, that these fluctuations and the diffusion that describes them occur similarly in all inertial reference frames.

It was shown that these two physical principles, together with the principles of mechanics, lead to diffusion quantum mechanics (DQM), which takes into account the fact that classical particles fluctuate and perform conservative diffusion not in empty space, but due to 
the interaction with a fluctuating background field. Moreover, the entire formalism of quantum mechanics naturally follows from DQM in the particular case of a homogeneous and isotropic background field.

The importance of starting from physical principles in such a fundamental theory as quantum mechanics becomes clear when they lead to clarity in the understanding of physical phenomena. Clarity of understanding gives confidence in the reliability of this theory, allows one to understand the prospects for its development and leads to new discoveries, while simplicity and visibility facilitate its teaching and popularization.

\subsection{The problem of the source of quantum fluctuations and the Munchausen paradox}

The mathematical formalism of quantum mechanics arose at trying to describe the quantum fluctuations of micro-objects observed in experiments. For an adequate physical treatment of this formalism, it was necessary to determine the source of fluctuations of microobjects - these are either devices, or they are themselves, or it fluctuates the surrounding background.

First of all, fluctuations due to devices were studied, since during measurements the influence of macroscopic devices on classical microparticles could systematically introduce an element of randomness. But it turned out that quantum phenomena are not explained by these fluctuations. First, in an ensemble of many identical experiments by a single particle, randomness due to measurements can be minimized and, nevertheless, the quantum properties of microparticles, for example, discreteness of energy levels or radioactive decay, do not disappear. Second, quantum phenomena determine the structure of matter in the entire universe without any observers and their instruments. Thus, it became clear that devices do not generate, but only express the objectively existing quantum fluctuations.

Then the sources of quantum fluctuations can be either the micro-objects themselves or the surrounding background. The standard formalism of quantum mechanics is based on the first possibility, and DQM consistently realizes the second.

The standard formalism is based on preserving the assumption of classical mechanics that the surrounding background is empty Euclidean space. But then micro-objects had to be ascribed some mysterious "quantum" properties - the ability to fluctuate spontaneously. In this case, the assumption of the classical nature of the background at the level of microparticles is only an extrapolation of the previous simplification and has no observational basis. And the result of this - the randomness of changes in the energy and momentum of microparticles in homogeneous space and time - is paradoxical and this is what caused many objections. Einstein was one of those who vaguely understood the inadmissibility of this and considered it a sign of the incompleteness of quantum mechanics.

In quantum mechanics, at spontaneous fluctuations with a sufficiently high energy, a particle has a finite probability to leave a potential well, breaking a barrier of any finite height and width. If one assumes that space is empty and that nothing but a locking potential acts on the particle, then a paradox arises, since the particle has nowhere to take the missing (and not small) energy. Explaining this by the uncertainty relation is a well-known logical trick, when the cause is explained by its own consequence, since this relation is a consequence of the presence of quantum fluctuations and cannot be their explanation.

An analogue of such a spontaneous fluctuation of energy among people is the paradox, which in [41] was called the Munchausen paradox. In a famous myth, this man once pulled himself from the swamp to the shore along with a horse, pulling himself strongly by own hair. The paradox is that he obviously didn't drown, but the way he got out was a miracle. To solve the paradox, it is important that the way Munchausen exits the swamp is not a confirmed fact, but the myth about the miraculous ability. Similarly, the choice of the particle that emerged from the potential well as the source of the energy it needs is not a fact, but the same myth about a miraculous (Munchausen's) ability that arose from the desire to preserve an empty 
surrounding background. But this desire does not justify the rejection of such a basic law of physics as the conservation of energy and momentum of an isolated object in a homogeneous and isotropic space.

In DQM, where the second possibility is used, when the particles are classical, and the source of their fluctuations is the action of the surrounding background, the Munchausen paradox is obviously absent. If, under the influence of fluctuations of the background field, the energy of a particle increases for a short time, then the energy of the background field in this place decreases to the same value, which ensures the conservation of the system's total energy.

The background field was introduced in a number of other treatments, but simplified models of fluctuations of this background did not allow them to become consistent theories. Nevertheless, they showed that an external fluctuating background field can be a more natural source of fluctuations of microparticles. In contrast to these simplified models based on specific models, DQM is based on physical principles following from observations and introduces a background field without specifying its specific properties. It takes into account only the facts of fluctuations of this field and conservation of the total energy in the system "particle + background field". In addition, the principle of relativity requires the invariance of fluctuations and diffusion in this field, which leads to the conservative character of diffusion regardless of its specific mechanism.

The tasks for further research in DQM are mainly the causes and mechanisms of fluctuations of the background field, as well as their further consequences. Acceptance of these fluctuations as an observational fact in the form of an appropriate physical principle leads to a simple and clear physical picture of quantum phenomena.

\subsection{Why in quantum theory the amplitudes of probabilities are summed?}

In classical physics, the probabilities of two alternatives are summed. In quantum mechanics, the probability density is defined as the square of the modulus of the complex amplitude (density) of probabilities, which for historical reasons is called the wave function. In this case, not the probabilities of the two alternatives themselves are added, but their amplitudes of probabilities. As a result, additional terms may arise that decrease or increase the sum of the probabilities of the two alternatives. An example of this is the probability interference pattern in an ensemble of particles passing through a screen with two slits.

In DQM, the state of an ensemble of classical particles is described by a probability density and a function related to the drift velocity of the particle flux. From these, the probability amplitude is formed in terms of coordinates and time. The canonical equations of motion for these two functions are nonlinear, but when a wave function is formed from them, these equations are linearized and turn to the Schrödinger equation, the basic equation of quantum mechanics. The linearity of the Schrödinger equation then leads to the fact that there is a superposition for the wave functions and therefore the amplitudes of the probabilities of the alternatives are added.

The reason for the difference from ordinary dissipative diffusion, where the probabilities are added, is the conservatism of diffusion in the background field, the conservation of the average energy of the ensemble of particles, i.e. lack of dissipation of its energy. The average values of physical quantities in DQM coincide with the average of quantum mechanics.

\subsection{Are there hidden parameters and what is the reason for nonlocality?}

Within the framework of quantum mechanics, several mathematical theorems were proved, which were considered as strong restrictions on attempts to interpret it in terms of classical concepts. The first of them was von Neumann's theorem on the impossibility of hidden parameters, and the second was Bell's inequality about nonlocality.

The first theorem really ruled out the theory of hidden parameters, in which certain internal degrees of freedom were attributed to quantum particles, which are not yet observed, but may eventually be discovered. The second theorem concerned the difference in the solution 
of nonlocality issues in classical and quantum theories, and quantum mechanics has been successfully confirmed in many experiments.

In this case, both theorems proceeded from the main difference between the classical and quantum theories, that in the first theory the probabilities are added, and in the second - the amplitudes of the probabilities. DQM turned out to be the only consistent theory that describes the phenomena in the same way as in the classical theory, but in it the amplitudes of the probabilities are added, just like in the quantum theory. For this reason, DQM is in the same group of theories as quantum mechanics and, being simply its more consistent formulation, satisfies the constraints of von Neumann's theorem and Bell's inequalities.

In this case, the reason for the nonlocality effects is that DQM describes the interaction with the background field not of an individual particle, but of an ensemble of particles, and at each moment the states of both the background field and the ensemble are defined globally.

Notice, that this theorem and inequalities refer to an isolated classical particle in an empty Euclidean space and correctly prove the impossibility in this situation of hidden parameters leading to spontaneous fluctuations (Munchausen's paradox) and nonlocality effects (violation of relativistic causality). But they do not belong to a classical particle in an external field and in this case they do not restrict anything. Therefore, the DQM, within the framework of which quantum properties arise because of such an interaction, is in agreement with these restrictions.

\subsection{What is the physical meaning of the uncertainty relations and quantum potential?}

The total energy of an ensemble of classical particles in empty space and an external potential is equal to the sum of the kinetic and potential energies, and in DQM, the energy of the diffusion flux in the background field is added to them. This diffusion flux arises when the probability density has an initial inhomogeneity and tends to equalize this inhomogeneity, i.e. directed towards the lower density area.

The physical meaning of the energy of this diffusion flow is that external forces (the walls of the box, external field, etc.) previously spent some energy in order to form this inhomogeneity in the distribution of the ensemble of particles in space, and now this energy is manifested in the form of the energy of the diffusion flow.

As smaller the area of localization, as larger the degree of inhomogeneity of the probability density and as more energy is required to localize the ensemble. This fact explains the physical meaning of the uncertainty relations, according to which as smaller the localization region, as larger the uncertainty of the momentum in the ensemble.

Therefore, the so-called "quantum potential", which appeared in the representation of the Schrödinger equations in the form of the Hamilton-Jacobi-Madelung equation, turns out to be the energy of localization of the ensemble of particles in a finite volume. The quantum potential as the energy of localization leads to the spreading of the ensemble of particles. Since diffusion delocalizes the ensemble of particles and, ultimately, equalize the probability densities, this stops the diffusion itself. Therefore, the quantum potential plays the role of the potential energy of localization.

\subsection{What is the physical meaning of the light velocity and its constancy?}

Diffusion of particles occurs due to their fluctuations in continuous media. Therefore, the main characteristic of particle's diffusion, which expresses the interaction with the medium, is the particle's fluctuation velocity. In a typical environment, the average value of this velocity is equal to zero, while the rms value is nonzero.

In classical statistical mechanics, a fluctuating medium in the form of a gas of atoms and molecules, has a rest frame where, for example, a box with gas is at rest. This frame of reference turns out to be the preferred frame for impurity atoms diffusing in a given gas. 
In the case of the background field, the principle of relativity excludes the existence of a distinguished frame of reference and requires that fluctuations of classical particles in this field occur similarly in all inertial frames of reference.

This is possible only if the value of the free run speed of the particle between interactions with the background field is an invariant velocity, which is the same in all inertial frames. Such velocity is well known in physics and represents the light velocity.

Thus, the light velocity turns out to be the velocity of fluctuations in the background field, and the constancy of the light velocity in all inertial reference frames, the property on which SR is based, is a consequence of the principle of constancy of quantum fluctuations in the background field. Transition from classical mechanics to relativistic theory, thereby also turns out to be one of the consequences of DQM, like SR itself.

Within the framework of the theory of relativity, there have still been questions that have not been answered: why is the light velocity universal and why is it invariant? Within the framework of DQM, as one can see from the above, answers to these questions arise: the light velocity is the velocity of fluctuations of classical particles in the background field, and it is invariant because quantum fluctuations are invariant.

Thus, instead of two postulates, about the invariance of quantum fluctuations and the invariance of the light velocity, in DQM one postulate about the invariance of fluctuations is enough, and the second, about the light velocity, turns out to be its consequence.

\subsection{What is the physical meaning of the rest energy of particles?}

The thermal energy of particles in a medium (gas, liquid) with a certain temperature is equal to the average kinetic energy of their thermal fluctuations, i.e. proportional to the mass and rms velocity of the particles. In DQM, the same situation, naturally, should be for finite mass particles in the background field, i.e. they must have "thermal" energy of fluctuations.

For an ensemble of free particles, the energy density of which is uniform in space, there is no localization energy. Therefore, if one excludes the drift energy for them, passing into the rest frame, then for such free particles the total energy is reduced only to their "thermal" energy in the fluctuating background field, which should be proportional to the particle mass.

In addition, as noted in the previous section, the velocity of "thermal" fluctuations of particles in the background field must be invariant and therefore equal to the light velocity. Consequently, the "thermal" energy must be proportional also to the square of the light velocity.

This part of the total energy of the particles should have appeared earlier both in theory and in experiments. At considering particles in empty classical space, this energy should have looked mysterious and inexplicable. This energy is well known from the relativistic theory and is the rest energy of the particle $E_{0}=m c^{2}$. Therefore, it should be identified with the constant thermal energy of fluctuations of a particle in the background field.

Thus, the energy of fluctuations of a particle of finite mass in the background field contains a constant part, the energy of "thermal" fluctuations, which manifests itself as the rest energy. Thus, if the relativistic theory introduced into physics the rest energy, a new addition to the energy of any particle of finite mass that plays a significant role in physics, then DQM allows one to explain its meaning and origin.

\subsection{How quantum statistics and particle indistinguishability arise?}

DQM studied the diffusion description of a state of an ensemble with one particle. The ensemble of experiments made it possible to determine the probabilities in many experiments, each time performed on one particle under the same conditions.

Classical statistical mechanics deals with systems of a very large number of particles, where the source of fluctuations is the randomness of collisions or other forms of interaction in a many-particle system. The phase space of a system of $N$ particles is divided into small cells and the probabilities are calculated in the intervals of particle's energy. In particular, the 
probabilities of filling of $M$ states with certain filling numbers are set by the number of ways (statistical weight) that these states can be filled with. In classical statistical mechanics, particles are distinguishable, and the probabilities of all states are considered as equal. Since the number of states is large, the average occupation numbers of states are very small. At low average occupation numbers, the particles are distributed over states independently of each other.

By placing each of $N$ distinguishable particles in one of $M$ states, one obtains a certain number of possible distributions. Since those of them that differ only by the permutation of particles are considered the same, then one must also divide this number by the total number of permutations. Thus, one finds the statistical weight of the distribution of $N$ particles over $M$ states, the inverse of which is the probability of realizing each of the distributions. From the requirement that the entropy at equilibrium be maximum, then the distribution function of particles of an equilibrium gas is found in classical statistics, which is given by the MaxwellBoltzmann (MB) distribution.

In the formalism of quantum mechanics, the probabilities of realizing different states of a system of particles were also considered as equal and the difference from classical statistics was reduced to two new properties: a) the particles were considered indistinguishable, and b) the filling numbers did not have to be small. It turned out that the assumption of indistinguishability of particles changes the statistical weight of states so that classical statistical mechanics goes over into one of two forms of quantum statistics.

The first of them refers to a system of particles with a wave function symmetric under permutations of two particles and in each state, as in the classical case, there can be any number of particles (Bose-Einstein (BE) statistics). The second refers to systems where the wave function is antisymmetric and therefore in each of the states there can be no more than one particle (Fermi-Dirac (FD) statistics).

In DQM, the particles remain classical and therefore remain distinguishable. Therefore, the question arises as to how quantum statistics, where particles are effectively indistinguishable, can appear in a system of classical distinguishable particles?

In DQM there are two types of probabilistic laws in a gas of microparticles - collisions of particles with each other lead to statistical laws similar to classical statistics, and conservative diffusion of particles between collisions leads to quantum properties of the system. The role of diffusion between collisions is insignificant at high temperatures and high density of states, when the occupation numbers are small, but it is significant at low temperatures and not small occupation numbers.

Therefore, if for classical particles in empty space all states are equally probable, then for the same particles that also fluctuate between collisions due to the background field, these probabilities do not have to be equal.

This distinction manifests itself most easily for states with antisymmetric wave functions, when there can be no more than one particle in one state. This property is not related to the many-particle nature of the system and holds even for a system of two particles, i.e. this is precisely the consequence of quantum fluctuations. Therefore, for such systems, instead of the classical number of distributions, it is necessary to take the number of combinations of $M$ elements by $N$, as a result of which the MB distribution is replaced by the FD distribution.

The reason why BE statistics appears in the system of classical particles was found by Tersoff and Bayer in 1979 [32]. It turned out that the condition of equal probability of different states of distinguishable classical particles, when the probability of a particle entering each of $M$ states is equal $1 / M$, is an unnecessarily strong constraint. By introducing a softer condition instead, the BE distribution was obtained in classical physics.

The softening of the conditions consisted in the fact that the probabilities can be arbitrary and only their sum should be equal to one, after which it is averaged over their admissible values. However, earlier the question arose here of why the equiprobability should be replaced by averaging over arbitrary probabilities and there was no answer to this, because, in the 
classical ideal gas, there is no reason for this, while in standard quantum mechanics, particles are not considered classical.

In DQM there is a clear answer to this question. Classical gas dynamics assumes that particles move between collisions rectilinearly in empty space. But in DQM, classical particles also interact with the background field, and additional fluctuations occur between collisions. Therefore, for each particle, the filling probabilities acquire an additional random scatter. Hence, it is necessary to take into account this new spread in probabilities and average over all these deviations. Then, in the gas of distinguishable particles, which additionally fluctuate between collisions, the BE distribution takes place.

Correlation between particles in different states, which is absent in classical mechanics, in DQM arises from the fact that particle's diffusion is described by globally defined probability amplitudes, and the amplitudes of a set of particles overlap. The indistinguishability of particles in quantum statistics also manifests itself effectively as a result of taking into account these additional fluctuations and the corresponding correlations between particles in their ensemble.

A fundamentally new property of quantum fluctuations is the Pauli exclusion principle for systems of particles described by an antisymmetric wave function upon rearrangement of particles. Without discussing the details of the relationship between spin and statistics, it should be noted that DQM, which describes processes using probability amplitudes, generally allows such states, regardless of the reasons for their occurrence. The probability density is the square of the modulus of the amplitude, and as a result, the amplitude, unlike the probability, can have both signs. Therefore, if, for some reason, conservative diffusion takes place with antisymmetric wave functions that change sign at permutation of particles, then all the consequences of the FD statistics and Pauli's principle will be observed, following from the change in sign of the wave function at permutations.

\section{Problems of quantum field theory and their solutions}

The problems of physics of particles and fields have mainly "technical" and they were solved without significantly changing the basic principles. The changes were reduced to clarification and more accurate formulations. The first five of problems were:
12. How to quantize the negative energy states correctly?
13. Are there zero-point fluctuations of vacuum fields?
14. How to quantize strings and do they have critical dimensions?
15. What are the consistent conditions of microcausality?
16. Why spin and statistics are related?

They turned out to be closely related, since the solution of the first problem solved the next two, and these two solutions led to the solution of the other two.

The next related problems of QFT are the questions:
17. Are high-order perturbation theory corrections infinite?
18. Are chiral anomalies infinite and is their cancellation in SM accidental?
19. Is quantum gravity consistent?
20. How to describe the possible substructure of fundamental particles?
21. Is there a need for supersymmetry?

To solve the first two problems, it turned out to be sufficient to take into account one of the basic gravitational effects of GR, which has been experimentally confirmed, but has not yet been taken into account in particle physics for purely historical reasons. And the answers to other questions follow from the solution of the above problems. These results are presented in articles [40] and in the book [46]. 


\subsection{How to quantize the negative energy states correctly?}

In QFT, the states of positive and negative energy arise on an equal right. In this case, negative energy particles from the very beginning were interpreted as a way of describing antiparticles of positive energy. But the realization of this idea turned out to be not easy and, after several stages, the problem was solved only recently.

In the naive approach, the negative energy states led to two major problems that made the theory inconsistent. The first problem is the continuous falling of particles into these states. Dirac's hypothesis [1] about a filled vacuum and holes in it solved this problem only for fermions and could not solve it for bosons. The second problem is the appearance of negative probabilities for negative energy particles in the standard quantization of fields [26].

The first problem was solved in the 1940s in the Stueckelberg-Feynman (SF) treatment [8,31]. This treatment introduces of particles of two signs of energy, where the negative energy particles, going backward in time only, in fact describe the positive energy antiparticles, going forward in time only. Then the transition of a particle to a state with negative energy means the process of annihilation of a positive-energy particle-antiparticle pair with the transition of their energy into radiation energy.

However, in the SF treatment, the second of the problems - the appearance of negative probabilities - was not solved, which made it inconsistent. For this reason, its application to QFT was considered as inconsistent and heuristic only.

As a result of this, the formulation of QFT with particles and antiparticles of only positive energy, when there are no problems with probabilities, has become standard. In this formulation, the creation operators of negative energy particles were replaced by the annihilation operators of antiparticles "manually". But, as a result of this manual operation, an infinite zero-point vacuum energy appeared even when it was absent there before this replacement. This meant that the theory also was inconsistent for this reason (this problem and its solution are discussed below).

Understanding of these facts led to attempts to return to the initial picture with particles of both energy signs, somehow solving two of their problems. From these attempts, a real step forward was the finding and elimination of the cause of the appearance of negative probabilities by M. Pavšič [27] in 1998. He started with an expression for the field energy operator with both signs and showed that in this case the probabilities of all states remain positive.

But both in this and in a number of other attempts, the negative energy states have been introduced in the hope that their zero-point energies would reduce the zero-point energies of the positive energy states. But for this they also had to be considered as going forward in time, which contradicted the SF treatment and thus the problem of falling into the negative energy states arose again.

In the first of the papers [40], a new method of consistent quantization of systems with states of two energy signs was formulated within the framework of the SF treatment, free from both problems of the negative energy states.

In the new method, on the one hand, all states are described according to the SF treatment, which excludes the fall of particles into the negative energy states. On the other hand, the description of the functions describing the trajectories of the particles, including the expression for the field energy, are initially chosen as strictly satisfying the SF treatment requirements. This condition for the internal consistency of the theory was not previously fulfilled, which was the mistake of previous attempts. The way to ensure the fulfillment of this condition was indicated in [40], after which it turned out that the SF treatment automatically reproduces the Pavšič's results [27] and all states have positive probabilities. Thus, in such a consistent formulation, the SF treatment turned out to be applicable in QFT also.

One of the changes in QFT formalism in the transition to the SF treatment was the introduction of a time-symmetric chronological products of operators, which turns into an ordinary chronological product at forward and an anti-chronological product at backward in 
time evolution. The time-symmetric chronological ordering operator naturally led to the SF causal propagator and to the standard diagram technique for interacting fields.

\subsection{Are there zero-point fluctuations of vacuum fields?}

In the standard formulation of QFT, the states of free fields have only positive energy, and the antiparticle operators are introduced "manually". As a result of such an introduction of antiparticles, an infinite energy of the vacuum of fields appears even in those cases when such energy was not present before the transition to antiparticles. This is the "zero-point energy" of the vacuum, the field, analogous to the "zero-point energy" of harmonic oscillators, the lowest energy level, and was considered as a manifestation of the "zero-point fluctuations" of the field.

The contribution of the zero-point fluctuations, which is infinite, was predicted for all SM fields, and this fact, due to the inevitable taking into account of gravity, made the theory inconsistent. In the presence of the zero-point energy, i.e. huge energy density at every point of the universe, instead of the observed extreme smallness of the effects of gravity in particle physics, its opposite would take place - the complete dominance of the effects of gravity, which is absurd. This situation has been called as the cosmological constant problem.

In the paper [40], where a new quantization method of fields and strings in the SF treatment was formulated, leading to the positive probabilities only, it was also shown that some well-known expressions for the energy of classical fields do not lead to the zero-point energy. This consistent quantization method was applied to harmonic oscillators with both signs of energy, and then to relativistic fields and strings. In this case, it is possible to pass from the SF treatment to antiparticles by using the charge conjugation or crossing symmetries.

There are a number of consequences of the new quantization method. In particular, the absence of the zero-point vacuum fluctuations is in agreement with experiments, since the effects attributed to these fluctuations (Lamb shift, Casimir effect) are fully explained by the contributions of the fields of real sources. The absence of the zero-point energy of free fields partially solves the cosmological constant problem.

Thus, the two results considered in last two sections, guaranteeing positive probabilities and the absence of the zero-point energies, improve distortions in field quantization procedures and make the QFT formalism mathematically correct and physically consistent.

\subsection{How to quantize strings and do they have critical dimensions?}

The string hypothesis suggests that fundamental objects of physics are strings, onedimensional objects of the Planck length order without thickness, and that their quanta are observed as known particles. It was assumed that the strings have critical dimensions of spacetime - 26-dimensional for a bosonic string and 10-dimensional for a fermionic one. Symmetries of these high dimensions then fixed the symmetry properties of strings and their quanta.

However, this purely speculative hypothesis and its generalizations, although they occupied a dominant position in particle physics in the last four decades, nevertheless, not only do not have an empirical basis, but, as shown in the paper [40], are inconsistent from a theoretical point of view also. It was shown that at a consistent formulation, string models will become trivial, since they will not have a distinguished space-time dimension.

The reason for this is that the standard formulations of the bosonic and fermionic string models included infinite zero-point energy of the vacuum, but since these models also claimed to describe gravity, it was impossible to remove the infinite part of the zero-point energy in them. The "regularization" procedure of the standard QFT, i.e. removal by hand an infinite part of the vacuum energy, made sense only in the absence of gravity and became illegal in models with gravity. Thus, the first string models were internally contradicting and inconsistent.

Attempts to overcome this contradiction have led to superstring models that contained an equal number of bosonic and fermionic degrees of freedom and therefore supersymmetry could be realized in them. In former quantization procedures, bosonic and fermionic degrees of freedom had the zero-point energies of opposite signs, and supersymmetry led to their mutual 
cancellation, although only in 10-dimensional space-time. But even in this case, supersymmetry had to be broken at distances of the order of the Planck length, which again gave rise to the problem of a huge vacuum energy density, incompatible with the existence of gravity. As a result, superstring models turned out to be far from reality.

However, the real situation turned out to be much simpler. At the consistent quantization of the relativistic strings in the SF treatment, there are models without zero-point energy, which are therefore finite and admissible from the physical point of view. However, in the absence of the zero-point energy, there were no critical dimensions of space-time. Thus, if the string models with zero-point energy were inconsistent, then the models without it became trivial.

\subsection{What are the consistent conditions of microcausality?}

The former standard formulation of QFT was based on the microcausality condition used in the classical theory of relativistic fields. It was argued that field measurements at one spacetime point (event) do not affect measurements at another point if they cannot be linked by a light signal. In quantum mechanics, this meant the simultaneous measurability of field variables at these points, which was expressed in the requirement that the operators of the boson field commute. The permutation functions (commutators) of bosonic fields, therefore, had to be odd functions of the time interval, disappearing behind the light cone, in which microcausality and locality in QFT were expressed.

Due to the direct replacement of the negative energy particle creation operators by the positive energy antiparticle annihilation operators, the commutators of the field operators were given by the Pauli-Jordan function vanishing behind the light cone. This was considered evidence of the fulfillment of the classical microcausality condition in QFT and was used in generalizations and applications of the theory.

At the same time, the causal propagator for field quanta did not disappear behind the light cone. In this case, the amplitudes of superluminal displacements of particles and their antiparticles mutually canceled each other and, as a result, the probabilities of real displacements disappeared. This meant that in QFT superluminal displacements of individual particles and antiparticles are allowed on the scale of their Compton length, and the mutual cancellation of these amplitudes is a manifestation of the many-particle nature of the processes in QFT. But if the propagator allows superluminal signals (albeit in a mild form, that is, in a small neighborhood), then the classical causality based on the complete absence of such signals is no longer valid. Namely, measurement at one point can influence measurement at another point even outside the light cone through virtual quanta carried by the causal propagator.

For fermionic fields, the inconsistency of the definition of microcausality in the previous interpretation turns out to be even more obvious. The anticommutator of fermionic fields was previously represented through the Pauli-Jordan function and it was argued that it also disappears behind the light cone and the definition of microcausality was extended to fermions. In fact, this representation is true only inside and on the light cone, but outside the light cone, where the Pauli-Jordan function disappears and the anticommutator of the fermionic field is nonzero, this representation is erroneous.

Thus, the previous definition of microcausality, declared as the disappearance of commutators or anticommutators of fields behind the light cone, was valid for bosons, but not valid for fermions, and also contradicted the properties of causal propagators.

The new quantization method based on the SF treatment [40] appears consistent in this issue, since it leads to even permutation functions (commutators and anticommutators) for both bosonic and fermionic fields. These functions do not disappear behind the light cone, but quickly fall off beyond the Compton length, and in this sense they behave in the same way as causal propagators.

Thus, in the new formulation of QFT, a unified formulation of microcausality conditions is given both for both types of fields, bosons and fermions, and for both types of basic field 
functions - permutation functions and causal propagators. Therefore, this condition of microcausality is consistent.

From a physical point of view, this means that behind the light cone, the classical conditions of locality and relativistic causality are satisfied not strictly, but approximately because of the correlations of the field functions on the Compton length scales. Therefore, they are fulfilled the better, larger the distance in comparison with this characteristic scale of nonlocality. At the same time, the softening of the conditions for classical microcausality for all quantum fields is not something new, since it was in a latent form in the previous formulation, but only for fermions, and in an explicit form it was present for causal propagators.

\subsection{Why spin and statistics are related?}

In the previous standard formulation of QFT, the proof of the spin-statistics theorem [25] was based on two conditions. In the particular case of free fields, this is the condition for the positivity of the energy of quanta with spin half, which is why anticommutators were introduced instead of commutators, and in the more general case of fields with interactions, this is the condition of microcausality, understood as the absence of superluminal correlations of events at different points of the field.

Fields with spin half are quantized within the framework of the SF treatment by introducing anticommutators, assuming that this is a fermionic field. But in this case, anticommutators do not lead to the exclusion of quanta with negative energy. This means that the appearance of anticommutators in a consistent QFT without diverging zero-point energy is not related to the sign of the energy of quanta.

In the previous standard formulation, the fact of linking anticommutators with the energy sign was an artifact of the "manual" replacement of the creation (annihilation) operators of negative energy quanta by the annihilation (creation) operators of antiparticles, which led to the zero-point energy. But in QFT, on the basis of the SF treatment, the former condition of microcausality is modified, becoming softer. In this case, the anticommutators of the spin-half fields behave in the same way as the causal propagators that do not disappear behind the light cone. This does not create problems, but only restores the internal consistency of QFT, since one-particle constraints are not required to be strictly observed in the many-particle theory.

Thus, the microcausality condition, which in QFT is actually not as strict as in the classical theory, is also not a basis for introducing anticommutators. In this regard, two facts should be taken into account.

The first is the fact that integer spin quanta are described by symmetric wave functions, since, for example, in a system with two pairs of electrons, each of the pairs has an integer total spin, and upon pairwise rearrangement of electrons, the general wave function does not change its sign. This means that the first part of the spin-statistics theorem, that the integer spin particles are bosons, is obviously proven.

The second is the fact that the half-integer spin particles within the framework of the SF treatment can, in principle, be described by both symmetric and antisymmetric wave functions, but experiments show that the second one is true, i.e. they are fermions. Thus, at this level of our understanding of QFT, the second part of the theorem on the connection between spin and statistics, that quanta with half-integer spin are fermions, is not a theorem that has been proven, but is a postulate based on conclusions from experiments.

\subsection{Are high-order perturbation theory corrections infinite?}

The main unsolved problem of the standard formulation of QFT was the problem of ultraviolet infinities, the unlimited growth of high-order corrections of perturbation theory at small distances or high fluctuation energies. In the terms of Feynman diagrams, they are given by loop diagrams, and the problem was the divergence of the integrals of the loop diagrams.

In practice, a cutoff (regularization) was performed at finite distances (and energies) when the corrections are still small and the perturbation theory series converge. The parts of 
the corrections growing at increasing of the cutoff energy, together with the "bare" masses and charges of the particles, should have given their measurable masses and charges. Therefore, the substitution them by the masses and charges known from the experiment was logical, and this procedure, called "renormalization", made QFT a finite and successful theory.

The problem was thus reduced to the question of what is unknown physical mechanism that makes the regularizations finite so that the corrections to masses and charges remain small. A long search for such a new mechanism based on radical hypotheses, however, was unsuccessful. The main reason for this was the hasty conclusion that all known phenomena have already been taken into account.

In the second of the papers [40] it was shown that the necessary mechanism existed initially and this is gravitational time dilation, one of the experimentally confirmed basic effects of GR. The standard formulation of QFT included quanta of very high energy, but did not take into account their gravity, and in attempts to take into account gravity, it was considered only as one of the fields. But according to GR, the external gravitational field of quanta also slows down the local proper times w.r.t. the world time $t$ of distant observers.

As a result, at the Planck length, which is the gravitational radius of Planck energy quanta, all processes freeze in terms of the world time and, therefore, do not contribute to the probabilities of processes of particle physics. The freezing of quantum fluctuations means an extremely strong redshift of frequencies up to zero, i.e. taking into account the effects of gravitation of quanta leads to gravitational regularization of the integrals of loop diagrams at the Planck length.

The nonlinearity of the fields increases gravitational effects, and hence freezing begin at even larger distances, which further suppresses the contributions of high energies. The finiteness of high-order corrections of perturbation theory at small distances also makes consistent the models of nonrenormalizable fields if the corrections are small over the Planck length. For gauge fields and quantum gravity, the invariant Planck cutoff of integrals gives upper limits for loop corrections, which appear as small and their perturbation series converge.

The first empirical evidence of the finiteness of the field theories is agreement with experiment of SM with a high accuracy. The predictions of QED are the most accurate and well tested. The agreement with experiments of QCD and electroweak theory is also very well. The smallness of the calculated masses, charges, and magnetic moments of quarks and leptons, where the bare values are determined from comparison with the observed values, means that the loop corrections are small and the perturbation theory converges, which indirectly confirms gravitational regularization required by GR.

In this case, quantum gravity became not only consistent, but also significantly simpler than expected, since it reduced to one-loop graviton contributions, while the high-order contributions appear as extremely small.

Thus, QFT became finite and consistent when it was adapted to the already known and well-proven principles of physics, the requirements of which must be taken into account initially. In this regard, there is no longer a need to search and test radical hypotheses, and this greatly simplifies and sufficiently heals the situation in particle physics. It is also important that the long initial stage of the formation of the QFT, when, despite numerous successes of the theory, its foundations were internally contradictory, can now be considered over.

\subsection{Are chiral anomalies infinite and is their cancellation in SM accidental?}

There is a special class of loop diagrams, the triangular diagrams with fermionic propagators, which grow linearly with the cutoff energy and lead to chiral anomalies. In the case of gravitational regularization, even being finite, the anomalous diagrams lead to very large contributions to the observed quantities.

Therefore, the criterion for the consistency of particle physics models is the mutual cancellation of anomalies in the set of diagrams for each observed process. In the SM, it takes 
place such cancellation of chiral anomalies, which was one of the main criteria for selecting quark models and gauge field models.

Gravitational regularization makes the situation with chiral anomalies in gauge field theories methodologically reasonable, since the theory operates with finite, albeit large and ultimately cancelling quantities.

Another situation is with chiral anomalies in phenomenological models involving pions consisting of a quark-antiquark pair. The introduced local fields and pion currents are effective and lose their meaning at short distances.

\subsection{Is quantum gravity consistent?}

Quantization of gauge fields and graviton fields in the weak-field approximation is reduced to quantization of two transverse physical states of vector or tensor fields. Therefore, the quantization of the photon field in the SF treatment is applicable in this case also, and taking into account the nonlinearity and internal symmetries of the fields does not change the main conclusion about the absence of the zero-point vacuum energy. Since the absence of the zeropoint energy of free fields follows from the presence of two energy signs states of relativistic fields, the interactions of quanta do not change this result.

In the case of gravitons, the effective coupling constant is weak up to the Planck distance, where gravitational radius of quanta becomes equal to their wavelength, which leads to a strong redshift of the fluctuation frequencies. A rapid decrease in the frequency of fluctuations at such distances due to gravitational freezing leads to small nonlinearity effects.

The conclusion that when graviton field is quantized in one reference frame, in the transverse gauge and in the weak coupling approximation, the zero-point vacuum energy is absent, remains valid for all reference frames and for all gauges because of the gauge and Lorentz invariance of the vacuum of the field.

The conclusion that the loop contributions are finite due to self-gravitational field is also true for quantum gravity, since gravitational time dilation is only strengthened by the quantum effects of gravity.

The dimensional coupling constant in quantum gravity leads to power-law contributions from graviton loop diagrams. However, in contrast to a scalar field, the effective dimensionless coupling constant is very small in most of the energy range up to the Planck energy, so that the loop contributions of quantum gravity, both logarithmic and power-law, remain small even in this region.

As a result, quantum gravity is not only finite, but also allows perturbation theory to be applied. Physical observables can then be represented as bare values and small quantum field corrections. Expanding in these effective coupling constants, the perturbation theory series converge.

\subsection{How to describe the possible substructure of fundamental particles?}

In the SM of particle physics, the number of fundamental particles, formally introduced as structureless, is large and amounts to several tens. However, it is possible that these particles can have an internal structure at very small distances, and this is even desirable. If the primacy of stable particles of the first generation of quarks and leptons can still be understood, then it is difficult to consider next two generations to be just as primary, differing mainly only in masses.

In this regard, composite models of known fundamental particles were developed, the subparticles of which began to be called preons, using other names for specific models. However, until now there were no signs of the presence of a substructure in quarks and leptons, and even more so in gauge bosons, and the proposed models were not consistent.

The main difficulty in these models was the mass paradox - a small region of localization of preons leads, due to the uncertainty relation, to their large kinetic energy, while the observed masses of SM particles are much smaller. This means that the binding energy of preons should 
almost compensate for their kinetic energy. But even in this case, the mass difference between the excited states would be very large, and therefore the second and third generations of fermions cannot simply be excited states of the first generation.

Attempts to combine the mass paradox with quantum mechanics or to circumvent it in various ways have significantly complicated the models, as a result of which interest in preon models practically disappear. Attention shifted to non-composite particle theories, which gave rise to more problems than they solved and led to even more complex sets of primary particles than even the SM itself.

DQM reanimates composite models of SM, since it contains a mechanism for the formation of composite particles of low mass, similar to the mechanism of chemical reactions between impurities. This mechanism removes the mass paradox, allowing one to "avoid" the uncertainty relation $[37,41]$.

If in a medium of heavy atoms two light atoms with different masses began to diffuse at a distance much greater than the mean free path, then the diffusion mechanism prevents them from coming closer together. To form an ensemble of composite particles, it is necessary to localize each of the ensembles of impurity atoms in a certain small volume, and this implies the energy consumption is as greater, as smaller the final volume of localization of these ensembles. However, if two particles at first accidentally were at a distance less than the mean free path and in a shorter time of travel were able to form a bound state (the atoms combined into a molecule), then this composite particle (molecule) diffuses in the same way as other atoms, differing only in mass.

In the papers [37, 41] it was proposed to use the same mechanism for the formation of composite particles of small mass in DQM and then this mechanism was applied to the simplest of the preon models - the rishon model. This mechanism practically removes the mass paradox by making the composite models of SM particles consistent and thus further simplifies the situation with the unification of fields.

Thus, the composite models of SM particles represent the most perspective direction in the development of particle physics and complement the models of unification of interactions. The success of composite models would significantly reduce the number of fundamental particles, including quanta of interactions, which would greatly simplify unification models and narrow the range of possible candidates for such models.

Gravitational regularization opens up new perspectives especially for composite models. At first, the composite character of the scalar boson is predicted, and, moreover, on the scale of distances that may be available for experiments in the near future. In this regard, the theoretical problems of composite models become most relevant and they will probably be solved soon.

Notice, that a closer attention to composite models and a focus on solving their problems will lead to the fact that further progress in particle physics will be associated with a reasonable perspective from the point of view of historical tradition - a decrease in the number of primary particles and a simplification of the models describing them, including models combining their interactions.

\subsection{Is there a need for supersymmetry?}

The possible existence of supersymmetry, a symmetry between bosons and fermions, allowing to solve the problems with infinities in QFT and unification problems in particle physics, has been a priority hypothesis for the last half century.

There were three expectations from this new symmetry: mutual cancellation of the zeropoint energies of bosons and fermions, partial cancellation of infinities in loop diagrams, and searches for simpler models of combining fields and particles.

The idea of the possibility of canceling the zero-point energies of bosons and fermions follows from the fact that the standard quantization of fermionic fields gives rise not to the usual zero-point energy, but to the zero-point energy of a negative sign. 
However, the successive SF quantization of fermions showed that in fact fermions in the relativistic theory do not have the zero-point energy at all. In the previous treatment, the (negative) zero-point energy appeared due to the fact that the operators of antiparticle were entered into the fermionic field functions manually. In the SF treatment, the transition to antiparticles is not necessary and therefore there is no zero-point energy either. And if one still goes into the picture of antiparticles, then this can be done in diagrams of specific processes using crossing or charge conjugation symmetry, which is usually done in calculations. But such a correct transition does not change the field energy precisely because of the indicated symmetries. Thus, of the three main arguments in favor of supersymmetry, one related to the zero-point energies has now turned out to be irrelevant.

The finiteness of some loop diagrams was also considered an advantage of supersymmetry. But for this, the masses of bosons and fermions had to be the same, and there would have to be superpartners of the particles. But the differences in the masses of the observed particles and the multiplication of the number of fundamental particles due to the need for super partners make this supersymmetry unrealistic.

\section{Problems of gravitational collapse and their solutions}

The effects of GR have been verified in many experiments and observations, but phenomena in extremely strong fields, such as the fields of stars experiencing gravitational collapse, are not only nontrivial, but have been observed only indirectly. As the result, a number of alternative treatments arose, which at first should had to answer the questions:

22. How to describe structure and evolution of an extended object in GR?

23. Could the surface of a collapsing star cross its gravitational radius and fall into the center?

The conventional paradigm was based on mathematical manipulations with local solutions. But from a physical point of view, the main thing is that parts of an extended object must coexist simultaneously. This means that the positions of its parts at any moment of time must be described on the hypersurface of simultaneity. Moreover, this hypersurface of simultaneity must be matched on the surface with the hypersurface of simultaneity of the external world, where the field of the star is given outside it.

Therefore, in GR, the physical picture of the structure and evolution of extended objects, such as stars and fields, ultimately must be described in terms of world time, marking these hypersurfaces. This fact leads to the solution of following problems of gravitational collapse:
24. How does the shell collapse and will it have a horizon and a singularity?
25. How a dust star collapses and what its structure will be?
26. How does a rotating star collapse and will it have an ergosphere?
27. Could a relativistic explosion of a collapsing star occur?
28. Do collapsed stars have a "hairstyle"?
29. Can two collapsed stars merge?
30. How supermassive collapsars differ from ordinary ones?
31. Is there a quantum evaporation of collapsed stars?

These results are presented in the papers [39, 43] and in the book [47].

\subsection{How to describe structure and evolution of an extended object in GR?}

In classical physics, time is absolute and the hypersurfaces of simultaneity of moving frames of reference are the same. Therefore, the form of an object also is absolute, and a spherical object can be described in a spherical coordinate system in any frame. 
In SR, time is relative and the hypersurfaces of simultaneity of different inertial frames differ. As a result, the ball is spherically symmetric only in the rest frame of the center, and in other inertial frames it becomes an ellipsoid flattened along the velocity. Therefore, in SR, a spherical coordinate system is appropriate for describing a spherical body only in its rest frame and is inadequate to the symmetry of the body in the moving frames.

At describing the structure and evolution of an extended object in GR, the positions of its particles also should be taken on the hypersurface of the simultaneity of the rest frame of its center of symmetry. Events on this hypersurface are marked by world time moments $t$. But events on the world line of each particle correspond to the moments of proper time $\tau$. Thus, these events are described in terms of two types of time, where each of them expresses only one of the two basic properties of physical time for a given object.

The description of evolution in terms of $\tau$, expressing the local rate of physical processes in a given part of the object, has long been considered as a standard one. But the rate of flow of proper times of particles slows down in the rest frame of the center of the object for two reasons - because of the movement of these particles, and also because of gravitational dilation of proper times w.r.t. world time $t$. Therefore, different values of $\tau$ correspond to simultaneous events in different parts of the object, which means that $\tau$ does not describe the entire object as a set of simultaneously coexisting particles.

The latter is realized only at describing in world time $t$, expressing the global simultaneity of events both inside and around the object. This assumes the presence of synchronized world clocks both inside the object and in its vicinity, showing for simultaneous events the same value of $t$ everywhere. The dilation of the standard clocks, comoving the particles, w.r.t. the world clock expresses the relativistic and gravitational dilation of processes with these particles.

Moreover, like the relativistic dilation of the proper time of an accelerated object, gravitational dilation is irreversible and absolute. This means that if the standard clock in the center of the star delays behind the same clock on the surface, and the latter delays behind the standard clock at a large distance, then the same ratio of the times of these three clocks will be observed in any frame of reference.

\subsection{Can the surface of a collapsing star cross its gravitational radius and fall to the center?}

In Newtonian theory, the answer to this question was obvious: if the mass of the collapsing star exceeds several solar masses, then yes, its surface will fall inside gravitational radius of the star and at superluminal speeds will quickly reach the center, where, together with the rest of the star's matter, it forms a singularity, an infinitely dense state.

In GR, there is a gravitational dilation of local proper times $\tau$ relative to world time $t$, the time of external world, and therefore the descriptions of the surface evolution in terms of two times, $\tau$ and $t$, are different. Thus, the situation here is not so obvious, and therefore two treatments of the collapse process in GR have arisen.

The first treatment also gave the same scenario as Newtonian theory, since in GR, the trajectories of particles on the surface are described in terms of $\tau$ by the same formula as in Newtonian theory. This led to the black hole hypothesis that dominated for half a century. The essence of this treatment consists in supplementing GR with the hypothesis that at describing events on the surface, one can ignore events in the outside world and consider $\tau$ capable of going independently of $t$ on and inside gravitational radius. For this reason, $t$ was considered as a "bad" choice for the time coordinate, and $\tau$ as giving the true picture.

The second treatment gives a negative answer to the above question taking into account the fact that in terms of $t$ the surface of a collapsing star freezes outside its gravitational radius, never reaching it. The reason for this is gravitational dilation of $\tau$ relative to the time of the external world expressed by $t$. Near gravitational radius, $\tau$ practically ceases to go w.r.t. $t$, 
which means freezing. This treatment ultimately led to the theory of frozars, which does not introduce any hypothesis into GR and only describes the picture of the complete freezing of the star's structure in terms of $t$.

The world lines of particles on the surface in terms $t$ describe each moment of their existence in the real world and therefore give a complete picture of the evolution of these particles, as well as the surface as a whole. The fact that the particles on the surface practically stop moving does not change anything in matters of their existence and being outside gravitational radius of the star.

Notice, that in the Schwarzschild field [29], describing gravitational field outside and on the surface of the spherical star, the radial component of the metric contains a singularity, i.e. tends to infinity when this coordinate tends to gravitational radius of the star. In this case, the time component of the metric tends to zero. Two above mentioned treatments interpret these facts in different ways.

In the black hole hypothesis, the emphasis was placed on the fact that at transition to local comoving coordinates on the basis $\tau$, SR is locally valid and there is no singularity. From this, it was then deduced that local observers easily cross gravitational radius just as it was in Newtonian theory.

The frozar theory $[39,43]$ goes further in analyzing the consequences of such transformations of physically measured quantities, including proper times and radial distances, emphasizing that there should not be any hasty conclusions. The fact is that GR introduces into this picture a number of limitations that are absent in Newtonian theory, and therefore to draw conclusions without taking them into account, as was previously practiced, meant going beyond GR, as a result of which the conclusions had nothing to do with GR.

The fact is that at transition to other coordinates, the singularity is only transferred either from some components of the metric to others (as in the transition from spherical to Cartesian coordinates), or from the metric to the coordinates themselves (as in the transition from global Schwarzschild coordinates to the coordinates of local observers). Therefore, it is possible to exclude singularities in the metric components by transformations of coordinates, but only at the cost of transferring the singularity to the coordinates themselves. This means that the limits of change of the new coordinates will contain new restrictions which must be indicated. An explicit singularity in the metric, after transferring it to coordinates, becomes implicit, expressed in restricted limits of the new coordinates. Therefore, if one does not indicate these new limits and illegally extrapolates the limits of the former coordinates to the new ones, then this is just a mistake, and not getting rid of the singularity.

The black hole hypothesis did not take into account the fact that in the infinite time interval of the external world, the corresponding proper time interval on the star's surface is finite. If we carefully take into account this fundamental difference between GR and Newtonian theory, then we come to the theory of frozars.

\subsection{How does the shell collapse and will it have a horizon and a singularity?}

The model of a thin dust shell is the simplest and exactly solvable model showing the main properties of GR collapse. In terms of world time $t$, the time of the outer world, the collapsing shell freezes outside its gravitational radius, its interior remains flat, and test particles inside it also freeze in those places where they were caught by the freezing process. The world lines of shell particles describe in terms of $t$ every moment of their existence in the real world and therefore give a complete picture of the shell evolution, exactly as for the surface of a star.

Irreversible freezing with respect to $t$ of all physical processes on and inside the shell due to gravitational dilation of proper times is the physical phenomenon stopping the shell collapse. This fact of dilation and subsequent freezing of local proper times w.r.t. $t$ is a key fact for understanding the process of collapse for other objects also. 
The fundamental physical phenomenon, the freezing of processes at strong gravitation in terms of the world time, the time of the external world, distinguishes the collapse in GR from the Newtonian one, where there is no such mechanism for stopping the processes. The presence in GR of gravitational freezing means that of two intervals of physical time, one, for proper time, remains finite even when the second, world time interval, tends to infinity.

The collapse of a thin dust shell leads to the formation of a hollow frozar, an object completely frozen due to its own strong gravity ("frozar" from "frozen star"). It consists of a shell, frozen over its gravitational radius, and an empty interior, where space is flat and where test particles are also frozen. A hollow frozar does not have an "event horizon", since the shell freezes before reaching the gravitational radius, and does not have a singularity in the center, since the inner region remains spatially flat and there are no shell's particles.

Earlier, by extrapolating the Newtonian scenario of collapse into GR, the formation of a hypothetical black hole was predicted with an event horizon at gravitational radius and the infinite density of matter (singularity) at the center, formed due to the fall of the shell there.

However, the evolution of the dust shell on the hypersurface of simultaneity, where its center rests, clearly shows the fallacy from the physical point of view of such extrapolation. One of the errors consisted in the naive statement that not only in Newtonian theory, but also in GR, the shell passes gravitational radius at the finite moment of $t$, but external observers allegedly cannot get information about this. In reality, the equations of motion of GR make it possible to construct the world lines of the shell particles for any finite moment, and at each of these moments the shell remains outside its gravitational radius (Fig. 1).

Thus, according to GR, the shell freezes in terms of $t$ not reaching its gravitational radius and becomes a place where nothing happens. This is not only for external observers, but also for those test particles inside the shell, which also froze, so that their world lines are parallel to each other and there will never be any fall to the center.

When the shell collapses, the conclusion about the formation of a hollow frozar instead of a black hole is not associated with a particular choice of coordinates, but remains valid for any other choice of physical coordinates expressing real distances and ratios of



Fig. 1. The worldline of a falling test particle in terms of world time $t$ and proper time $\tau$. intervals of physical times on the shell and far from it. The physical distances between the shell particles, as well as the positions of the test particles taken at the same moment, will be the same for all frames of reference and will remain practically unchanged at subsequent moments of $t$.

\subsection{How a dust star collapses and how its structure evolves?}

A star as a whole, as an extended object, is a set of simultaneously coexisting particles, and therefore its structure in the rest frame of its center of inertia must be defined as a set of simultaneous events.

This definition, which is natural in SR, does not change in GR. For ordinary stars, including white dwarfs and even neutron stars, gravitational time dilation is small. In the general case, outside a spherical star, its gravitational field is static, and in static fields the simultaneity of events is determined in the same way as in SR. The same applies to the surface of such a star, where the outer and inner field solutions are matched. 
For the inner layers of a star, there are exact solutions of GR equations for simple cases, which make it possible to relate the proper times of particles in layers with the proper times on the surface. By selecting along the world lines of particles of layers those values of their proper times $\tau$ that correspond to events simultaneous with the surface, it is possible to describe the instantaneous structure of the star at the corresponding moment of world time $t$.

The structure and evolution of the star in this physical picture are determined from the analysis of exact solutions of the field equations and equations of motion with physically correct initial and boundary conditions. In the case of a spherical dust star, the density of which is locally uniform and the particles fall radially with parabolic velocity, the exact solution was found by Oppenheimer and Snyder (OS) in 1939 [23]. They transformed the well-known Friedman local solution (in Tolman's form [33]) in terms of $\tau$ to global solutions in terms of $t$. The OS solution made it possible for the first time to describe the structure of a collapsing star as a set of simultaneous events.

Later, exact solutions were also found for elliptical velocity by two other methods by $\mathrm{O}$. Klein in 1961 [17] and S. Weinberg in 1972 [34]. A complete solution to the problem of the collapse of a homogeneous dust star in GR by these three methods (OS, Klein and Weinberg) for three special velocities (parabolic, elliptical and hyperbolic) was given by the author in 2017 $[38,39,43]$. Note that these three velocities, preserving the uniformity of density, correspond to three values of curvature in homogeneous space - flat, positive and negative curvature.

It was shown that, not only the surface of the star freezes in terms $t$ above its gravitational radius, but also its entire volume too. During the collapse, the star's center freezes before the other layers, after which the entire structure of the star quickly freezes. This means that gravitational time dilation is the physical mechanism stopping the stellar collapse in terms of $t$. Each of the inner layers freezes near its effective gravitational radius created by the matter inside this layer (taking into account the influence of the outer layers).

Before freezing, the density of sufficiently massive stars is lower than that of a neutron star, and therefore, if they did not explode before, then the collapse of such stars occurs similarly to a dust star with the formation of a completely frozen star or a frozar. Thus, the collapse of

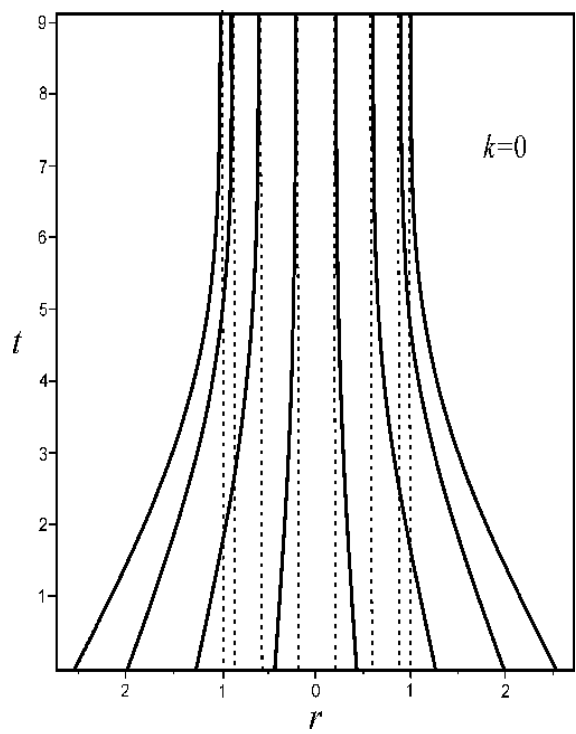

Fig. 2. World lines of stellar particles along the diameter (in units of the gravitational radius) according to the OS solution. At large $t$, particles on the surface freeze outside the gravitational radius, and the inner layers freeze near their asymptotes (dashed lines).



Fig. 3. OS worldlines from Fig. 2 with a slightly more number of layers to show how different layers freeze. 
stars in GR at any finite moment of $t$, the time of the external world, leads to frozar, an object that has an almost homogeneous and practically frozen internal structure [38, 39,43].

The plots of world lines of star's particles show the internal structure of the dust star at different moment of $t$ (Figs. 2-3). The world lines of particles in layers approach their asymptotes at the internal effective gravitational radii, which are parallel to the world time axis. Layers, which are equidistant at the beginning, will also remain almost equidistant in the lower layers during freezing and are compressed near the surface. This shows that the frozen star picture refers both to the surface, asymptotically approaching gravitational radius, while remaining outside it, and to the entire structure of the star.

Note that the Schwarzschild coordinates are physical coordinates in a static field, which adequately take into account the symmetries of the field and are measured by standard scales along circles and synchronously running everywhere world time clocks. Therefore, the introduction of other parameters instead of them does not change the physical results, since the description of physical processes and the structure of objects ultimately comes down to measuring the same actually measurable physical coordinates of particles. New coordinates, usually just some parameters, which are often impossible to measure. To obtain physically measurable values, one must return to the measured physical coordinates, the simplest of which are precisely the Schwarzschild coordinates. Therefore, the image of the structure and evolution of a star with asymptotically freezing layers on the hypersurfaces of simultaneity will be reproduced in all frames of reference with physical coordinates.

Due to the difference between the two forms of physical time in GR, proper time $\tau$ and world time $t$, there were attempts to continue the description of mythical "events" in the nonphysical area "outside the infinite world time." At the same time, the surface of the star was given the mythical ability to cross gravitational radius in the epoch "after infinity" and reach the center in a finite interval of proper time, forming a singularity. This area of mathematical physics became known as the black hole hypothesis and was widely promoted. However, it has nothing to do with real stars in the physical world, which exists only on the hypersurface of simultaneity with a finite value of $t$, the time of the surrounding external world.

Unlike neutron stars, when matter falls on a frozar, there will be no flash of radiation, since near the surface the matter and the process of its fall quickly freeze. Therefore, for an outside observer, the fall of matter on the frozar will be very "quiet". This and a number of other properties of frozars are consistent with the observed properties of well-known compact objects that are "candidates to frozars."

Thus, the collapse of stars in GR on hypersurfaces of simultaneity is sufficiently different from the scenario of the Newtonian theory. When the surface of a collapsing star approaches its gravitational radius, all processes in the star quickly freeze and the star will become frozar. Frozar is a star with a surface outside gravitational radius, the internal structure of which is completely frozen in the state in which it was before freezing. Frozars have no horizon and no singularity and lead to a number of observable effects, and the area of space they occupy is "a place where nothing happens.".

The hypothesis of black holes, the predictions of which have never been observed anywhere, is based on the Newtonian scenario, purely formally introduced into GR, and has nothing to do with the physics of real phenomena since are based on the assumptions that are natural in Newtonian theory, but unrealizable either in GR or in nature.

\subsection{How does a rotating star collapse and will it have an ergosphere?}

The space-time interval between two events around and on the surface of a rotating star is given by Kerr's solution [16]. This solution includes not only gravitational radius, but also the angular velocity and the parameter characterizing the flattening of the star due to rotation.

The proper time of a particle on the star's surface is dilated w.r.t. $t$ both gravitationally and kinematically. The non-radial part of the velocity of these particles is maximal at the 
equator and vanishes at the poles. The local velocity of surface particles only asymptotically approaches the light velocity at any finite moment $t$.

As the star contracts, the particles on the surface near the poles, as in the case of a spherical star, freeze as they approach gravitational radius. But at other latitudes, the total velocity of particles on the surface grows faster than at the poles, since the velocity of rotation is added to the radial velocity of falling. Therefore, the distances from the center, when the total velocity tends to the light velocity and the proper times of the particles freeze, will be more and more. At the equator, this distance, depending on the angular velocity, will reach its maximum.

Thus, for the external static frame of reference, as the rotating star contracts, the processes on its surface freeze in terms of $t$. Thus, the surface freezes, remaining an oblate ellipsoid. All processes in the inner layers also freeze, and the star turns into a frozar with angular momentum.

Gravitational time dilation in the layer of a star (in a static frame), like in a spherical star, is determined by the effective gravitational radius at the level of this layer. The latter depends both on the internal gravitational radius and on the time dilation effect created by matter outside this layer. In this case, the angular momentum of the rotating star is conserved, and its external field remains the same as before freezing, since freezing does not mean an absolute stop.

Thus, in a static frame of reference, the surface of a frozar with angular momentum ceases to shrink radially, remaining outside the outer gravitational radius of the Kerr metric, which was previously considered as the outer boundary of the ergosphere. The meaning of this boundary is that here the local speed of surface particles would reach the speed of light. Since the metric inside such a frozar is given by a material solution and is not the Kerr metric, such a frozar does not have an ergosphere. The direct result of this fact is the absence of effects associated with the possible existence of the ergosphere, such as the extraction of rotational energy when particles enter the ergosphere by subsequent release.

The previous extrapolation of the Kerr solution, which is a vacuum solution, into the interior of a collapsing star, had no logical basis and was only a non-physical mathematical abstraction.

\subsection{When a relativistic explosion of a collapsing star can occur?}

At considering the contraction of cold matter (dust or with pressure), the temperature effect was not taken into account, which led to gravitational freezing of the star with a transition to the frozar state. If temperature effects are taken into account, the contraction becomes hot.

Consider first the case of a supermassive star. In this case, when the stellar surface approaches gravitational radius, the average density still remains small and the equation of state of an ideal gas is locally valid. At the same time, the local temperature near the surface in world time grows rapidly.

The energy of the falling layer near the surface in the local static frames along its path increases. If, in the case of dust, this growth only increases the kinetic energy of the falling layer in each of these local frames, then in gas a part of this kinetic energy transforms into heat and the layer's proper temperature increases. In any case, near the center of such a star, the local temperature grows faster than on the surface. As a result, the local pressure near the center also grows rapidly.

Thus, as a low-density supermassive star contracts, exponentially fast increase (in world time) the local temperatures inside the star, faster than on the surface, which leads to the inhomogeneous increasing of local pressures. Local pressures near the surface change little, while local pressures in the vicinity of the center grow exponentially quickly (in world time), which at some stage leads to the stopping and the expansion of the inner layers of the star close to the surface, and then to the expansion of the surface.

We note a new and nontrivial relativistic effect, which consists in the fact that at the first stage there is an increasing concentration of the main mass-energy of the star near its surface 
with the formation of a semi-empty central and middle regions and a massive surface shell of the star $[20,39,43]$.

Due to the rapid rise in temperature, the stellar matter passes into a hot radiationdominated phase. The transition at first of the central and then of the upper layers to such a phase can stop the compression and lead to a relativistic explosion. There is a lot of evidence in the literature that in GR at numerical simulations by using realistic equations of state, a star can turn to the expansion state.

As greater the mass of the star, as more likely that freezing will occur faster than heating and a frozar will form. But as less the mass of the star, as more likely that the freezing will not be fast enough, and heating will stop the contraction and can lead to an explosion.

During contraction, a significant part of the matter will be near the surface, where, according to GR, the physical volume grows sufficiently, and the contraction energy is transformed into heat with the transition of the matter into a radiation-dominated phase. If the star did not have time to freeze, then part of the ultrarelativistic matter and radiation begins to quickly leave the star, which manifests itself as a relativistic explosion, and the object itself is observed as a relativistic supernova or hypernova.

\subsection{Do collapsed stars have a "hairstyle"?}

The surfaces of white dwarfs and neutron stars do not contain large irregularities due to their strong gravity. Any matter falling on them is also absorbed by a compact star or distributed over its surface.

In the case of frozars, the situation is the opposite due to the extremely strong gravity: the accreting matter, falling almost radially, quickly freezes on the surface, the transverse size of the accreting area of matter will not increase. As a result, the matter becomes radially flattened without spreading over the surface.

This leads to inhomogeneous distribution of the matter falling on the frozar's surface, similar to massive anomalies (mascons) on the lunar surface. When matter falls from the disk, the inhomogeneity is maximum near equator.

In particular, at radial falling of neutron star on a more massive frozar, the neutron star's matter will flatten and freezes on the frozar surface in the form of a local "hairstyle", a large mascon. This leads to an evident local inhomogeneity of frozar's gravitational field (Fig. 4), which will contribute to the variabilities and changes in the brightness of the disk around the frozar. It is also possible for a neutron star to explode due to transverse compression at falling on the frozar, which can lead to the observed powerful burst.

Thus, the formation of an inhomogeneous landscape on the frozar surface distinguishes frozars from two other classes of compact objects - white dwarfs and neutron stars. The observational effects of such a landscape associated with local anomalies of the frozar's gravitational field are a new field of research for studying the structure and formation history of each frozar [43].

The inhomogeneity of the density distribution of matter on the frozar surface leads to small inhomogeneities of the gravitational field over these areas. These inhomogeneities, although they decrease with distance, lead to the observed effects. In some

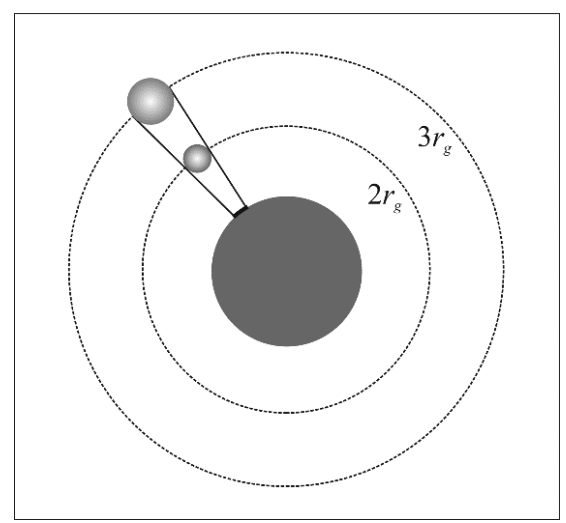

Fig. 4. The radial fall of a neutron star of the mass $1.5 M_{\circ}$ onto the frozar of mass $7 M_{\circ}$, which leads to flattening of the neutron star (like a mascon on the Moon) and freezing on the frozar surface like a "hairstyle". This leads to local inhomogeneity of the frozar field and its shadow. 
cases, for example, in the presence of orbiting sources of radiation, they can be detected using gravimetric methods, as well as irregularities in the redshifts of matter around the frozar.

One of the observed consequences of such perturbations of the metric is the appearance of small deviations in the shadow of a frozar. The gravitational deflection of light rays is distorted by metric perturbations. As greater the deflection of the rays from a more massive area of the surface, as increases the border of the shadow above that area, and the border of the shadow will contain irregularities.

Another class of observed effects is associated with the disturbance of redshifts and orbital trajectories of objects around a frozar. Perturbations of the metric can also lead to additional orbital precession, which can be observed from the radiation of the revolving object.

Large inhomogeneities on the surface lead to the appearance of inhomogeneities in the disk around the frozar, which contribute to the variations in its brightness and in some cases it may be possible to separate them from other contributions, especially for supermassive frozars.

\subsection{Can two collapsed stars merge?}

In Newtonian theory, two compact spherical objects of stellar mass or more at merging form a more massive compact object, the final stable state of which is also mainly spherical. However, in GR, two or more frozars cannot merge at approaching and freeze at some distance from each other, forming a frozen cluster of two or more frozars.

Let two frozars of equal mass and surface radius, very close to their gravitational radii, fall radially to the common center of inertia, where the center of the static coordinate system with world time $t$ is located. The gravitational radius of the system is twice gravitational radius of each of the frozars. At falling, the radial coordinate of the center of each frozar remains outside gravitational radius of the system due to the freezing of all processes, including falling, in terms of $t$. Thus, in GR, two frozars cannot merge, and it occurs their sticking together only. The same situation, even more illustrative, takes place for three frozars (Fig. 5).
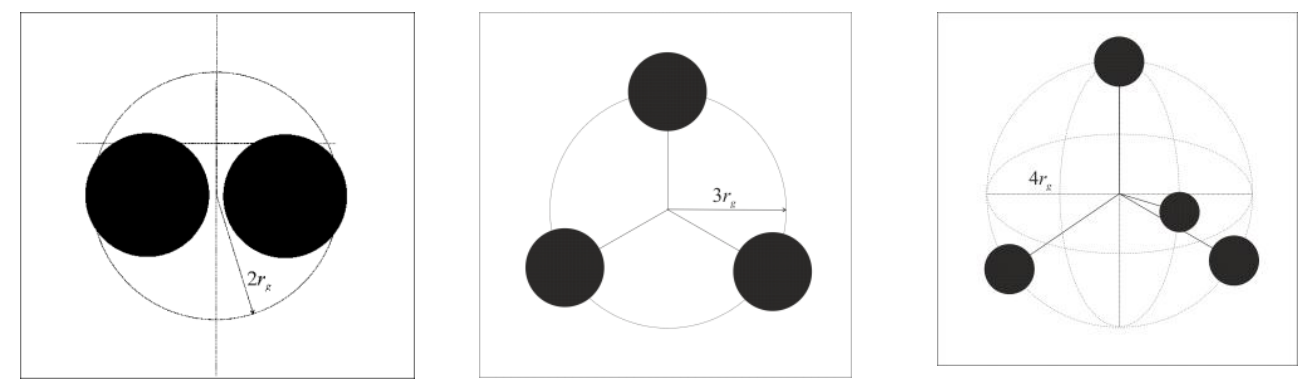

Fig. 5. Gravitational freezing of two, three and four frozars of the same mass on gravitational radius of the center of inertia of the system $n r_{g}(n=2,3,4)$.

This fact is especially clear in the case of four radially falling frozars of the same mass located at the vertices of a tetrahedron. Here gravitational radius of the system is about four times gravitational radius of any of the frozars (Fig. 5). In fact, the frozen cluster forms a gravitational crystal, where the distances between the frozars are several times larger than the sizes of each of them.

The result of sticking together of frozars represents the largest inhomogeneity in a frozar cluster. These inhomogeneities also lead to the observed effects that are stronger than the similar effects discussed above, such as mascons on the frozar surface. Gravimetry, as well as other methods related to studying the behavior of orbits and disks, can also allow studying the internal structure of such clusters of frozars. 
In addition, the sticking together of two frozars also results in a shorter and weaker burst of emitted radiation in the final stage, including gravitational waves, than at merging. This subject requires further study with detailed numerical modeling of the process.

\subsection{How supermassive collapsars differ from ordinary ones?}

Growth of frozars or their clusters during accretion with inhomogeneous freezing on some parts of the surface leads to the formation of a hybrid of frozars with ordinary matter. This process can lead to the formation of intermediate mass frozars, as well as supermassive frozars. As a result, such gravitationally frozen clusters of very large mass form a new state of matter, which apparently initiated the formation of galaxies in earlier epochs or was formed later due to the fall of stars and gas into the centers of galaxies.

In any of these cases, gravitationally frozen objects, sufficiently exceeding the solar mass, have a complex and inhomogeneous structure. This is due to the fact that, during their formation, falling massive objects, including entire stars, were destroyed by tidal forces only partially, and most of their matter was flattened and frozen in compact areas of the surface.

Usually, when an ordinary star is destroyed by the tidal forces of a stellar mass frozar, an accretion disk is formed around this frozar. In the case of supermassive frozars, where the tidal forces are small, ordinary stars and compact objects of stellar masses can be captured without losing the main part of their matter and then at the final stage they fall almost radially, forming a stellar mass mascon above gravitational radius of the system.

Thus, the fall of ordinary matter for a long time turns frozar clusters into a cluster of frozars and ordinary matter, since the falling matter also freezes on the cluster's gravitational field. Therefore, at modeling the structure of compact gravitationally frozen massive or supermassive clusters of frozars, it is necessary to take into account the formation of gravitationally frozen inhomogeneous clusters of frozars and ordinary matter.

The inhomogeneity of the structure of a supermassive frozar leads to perturbations of the gases rotating around it, which can be an origin of turbulence and oscillations that cannot be explained by the usual reasons.

\subsection{Is there a quantum evaporation of collapsed stars?}

At quantum fluctuations of fields in a static gravitational field, the worldlines of particles are ordinary both inside and on the surface of the star. In this case, the energy of the virtual particle-antiparticle pair is positive. Therefore, in GR, there is no quantum production of real pairs of particles from vacuum.

In the black hole hypothesis, the "quantum evaporation" of these black holes was predicted, since if one of the particles is born inside the "event horizon" (gravitational radius), then its energy will be negative and its fall to the center will reduce the total energy of the hole, and the second particle outside the horizon with positive energy could escape.

The absence of an "event horizon" in the correctly solved problem of collapse in GR, when the surface of a star freezes forever outside gravitational radius, and the "horizon" is never formed, makes the picture of "evaporation" meaningless as well.

However, in the case of vacuum fluctuations of quantum fields in the vicinity of a frozar, one of the quanta of the pair can fall on the frozar, and the other can leave its vicinity, if this is allowed by the conservation laws (for example, in the presence of a thermostat or external fields). In this case, the freezing of some of the quantum fluctuations in the frozar leads to the appearance of effective entropy and temperature.

But, in contrast to the hypothesis of "quantum evaporation" of collapsed objects, at such a production of particles due to the energy of external sources, the frozar's mass grows due to the absorption of positive energy particles. Therefore, in these cases, we should talk not about "evaporation", but about "condensation" on the frozar of the remnants of pairs of particles. 


\section{Problems of the theory of gravity and their solutions}

GR, almost all of the predictions of which were confirmed by experiments, gives a geometric theory of gravity, where gravity is the space-time curvature arising due to the presence of matter. But GR does not explain this fact and only proceeds from this, and therefore, behind this geometrical picture there must lie a certain physical mechanism explaining why matter curves space-time.

As the result, in the foundations of the theory of gravity, a number of problems remained unsolved, some of which will be considered below:

32. Was Maxwell right about the nature of gravity?

33. What underlies the principle of equivalence?

34. What is the physical mechanism of gravity?

35. What is the physical meaning of Einstein's equations?

Solutions to these problems are presented in the papers [41] and in book [45].

\subsection{Was Maxwell right about the nature of gravity?}

In the Maxwell's book "A Dynamical Theory of the Electromagnetic Field" (1864) [21] there is a very remarkable section "Note on the Attraction of Gravitation" (at the end of Part 4) with his hypothesis on the physical mechanism of gravity.

"After tracing to the action of the surrounding medium both the magnetic and the electric attractions and repulsions, and finding them to depend on the inverse square of the distance, we are naturally led to inquire whether the attraction of gravitation, which follows the same law of the distance, is not also traceable to the action of a surrounding medium.

Gravitation differs from magnetism and electricity in this; that the bodies concerned are all of the same kind, instead of being of opposite signs..., and that the force between these bodies is an attraction ...

The lines of gravitating force near two dense bodies are exactly of the same form as the lines of magnetic force near two poles of the same name; but whereas the poles are repelled, the bodies are attracted. Let $E$ be the intrinsic energy of the field surrounding two gravitating bodies $M_{1}, M_{2} \ldots$ If $R$ be the resultant gravitating force, .. hence $E=C-\sum R^{2} d V / 8 \pi$. The intrinsic energy of the field of gravitation must therefore be less wherever there is a resultant gravitating force.

As energy is essentially positive, it is impossible for any part of space to have negative intrinsic energy. Hence those parts of space in which there is no resultant force, such as the points of equilibrium in the space between the different bodies of a system, and within the substance of each body, must have an intrinsic energy per unit of volume greater than $R^{2} / 8 \pi$, where $R$ is greatest possible value of the intensity of gravitating force in any part of the universe.

The assumption, therefore, that gravitation arises from the action of the surrounding medium in the way pointed out, leads to the conclusion that every part of this medium possesses, when undisturbed, an enormous intrinsic energy, and that the presence of dense bodies influences the medium so as to diminish this energy wherever there is a resultant attraction.

As I am unable to understand in what way a medium can possess such properties, I cannot go any further in this direction in searching for the cause of gravitation."

Thus, even more than a century and a half ago Maxwell noticed that if the Newtonian potential refers to a field, then the energy density of this gravitational field is negative. Since he considered it unacceptable from the point of view of physics, he came to the conclusion that this fact says something about the physical mechanism of gravity. Explicitly, he predicted that a reasonable way out of the situation would be only in the case when in the whole space there is a certain medium with an enormous reserve of positive energy, greater in magnitude than the 
deepest of the "gravitational potential wells". Then the energy density of gravitational field expresses the deficit of the local energy density of this medium.

His main conclusion that "... each part of this environment, being unperturbed, possesses enormous internal energy and that the presence of dense bodies affects the environment in the direction of decreasing this energy", is in accordance with DQM based on the existence of a background field. As shown in [41] and will be considered below, the problem of the negativity of the Newtonian field energy, which caused Maxwell's concern, is naturally solved in the theory of diffusion gravity, which follows from DQM, and exactly as Maxwell assumed.

\subsection{What underlies the principle of equivalence?}

A complete explanation of the physical nature of gravity must include taking into account relativistic effects. The diffusion treatment of gravity in DQM therefore will consist of a combination of quantum and relativistic phenomena.

But as a first step, let us consider a simple case when inhomogeneities of the background field energy density are small and constant in time. These two simplifications allow one to derive from the DQM those basic physical properties of gravity, from which both previous theories, Newtonian and Einstein's, proceeded. These consequences of DQM should manifest themselves even at the level of classical physics, i.e. after averaging over quantum fluctuations and, therefore, the consequences of DQM will be analyzed in the framework of classical mechanics with addition of the lowest order relativistic corrections.

In DQM, the light velocity $c$ is the velocity of fluctuations of particles in the background field, and the energy of fluctuations of a particle, or its "thermal energy", equal to the rest energy $m c^{2}$, is the "energy level" occupied by the particle in the background field. Fluctuations of particles near a massive body will be slower than at far from it, which means that the velocity of fluctuations near the body $c^{\prime}$ is less $c$. As a result, $c^{\prime}$ becomes dependent on the distance to the massive body, and the rest energy of the particle near the body, respectively, turns out to be equal $m c^{\prime 2}$.

This decreased rest energy can be expressed through the "unperturbed" rest energy $m c^{2}$ as: $m c^{\prime 2}=m c^{2}+m \varphi$. Here $\varphi=-\left(c^{2}-c^{\prime 2}\right)$ and this negative-defined function plays the role of the "potential" for a particle in an effective external field, in the field of dilated fluctuations of particles in the vicinity of a massive body.

Thus, the change in the state of the background field near a massive body is reduced in the first approximation to the appearance of the potential energy of interaction of a particle with an effective external field, which is proportional to the mass of the particle and the potential energy $U=m \varphi$ in this field of dilated fluctuations.

The total energy of a particle with a drift velocity $\mathbf{v}$ then becomes the sum of the rest energy $m c^{2}$, kinetic energy and potential energy $m \varphi$. As a result, the equation of motion in the Newtonian approximation takes the form: $\mathbf{a}=-\nabla \varphi$. Here $\mathbf{a}$ is the acceleration of the particle due to the difference in the fluctuation velocities at nearby points of the background field. Since the mass of a particle drops out of the equation of motion, then, consequently, all particles in the effective potential $\varphi$ have the same acceleration, regardless of their masses.

This consequence of the diffusion treatment of gravity in DQM expresses the main distinctive property of gravity, which is absent in other fields. The principle of equivalence, formulated by Einstein on the basis of this property, led to an analogy with the behavior of particles in accelerated frames of reference and further, within the framework of GR, made it possible to discover the geometric nature of gravity [4]. 


\subsection{What is the physical mechanism of gravity?}

In continuous media, when a cold compact body is immersed in them, a fairly rapid heating of this body up to the temperature of the medium in this area occurs. This heating of the body leads to local cooling of the medium relative to other areas.

The result of local cooling of the medium, before its relaxation, is a decrease in the thermal energy of molecules in this region, which reduces the intensity of fluctuations of all molecules, both the medium and the impurity, in comparison with other regions with the same temperature. The appearance of a temperature gradient in the medium leads to a heat flux, as well as to a thermal diffusion flux of impurity molecules.

In DQM, at a high concentration of classical particles embedded in the background field, similar processes should occur. The intensity of particle fluctuations in this region should decrease due to a local decrease in the energy density of the background field, and this should lead to a thermal diffusion flux of other classical particles into this region with a lower intensity of fluctuations.

However, now it is not necessary that gradient of the energy density of the background field leads to a "heat flux" to a "colder" region and to an equalization of the local energy densities. In this case the dilation of fluctuations means also dilation of the local rate of time, since all processes slow down here. And this leads, as in GR, to two types of temperature - in terms of local proper times and in terms of world time. In this case, thermal equilibrium occurs when the temperatures are equal in terms of world time, while local temperatures can be different in different places.

In DQM, the conservative (average energy-conserving) diffusion of classical particles in the background field at its uniform energy density explains the formalism of quantum mechanics. In this case, a physical explanation is also given to two fundamental facts fluctuations in the energy of particles in the background field ("thermal" energy) is manifested as their rest energy, and the corresponding decrease in the energy of the background field around the particles is identified with gravity.

In DQM, the total energy of the "particle + background field" system is conserved. Since the fluctuations of a particle put away a part of the energy of the background field, which is equal to the rest energy of the particle, the energy of the background field itself in the vicinity of the particle becomes, on average, less to the amount of the rest energy of this particle.

The influence of one particle on the background field is insignificant, but a very large number of particles in a small region noticeably decreases the local energy density of the background field. This reduces the velocity of particle fluctuations here and leads to a thermal diffusion flux of particles into this region. Therefore, the potential of gravitational field is proportional to the mass of the source.

The velocity increments, due to the conservativity of diffusion, are cumulative, i.e. accumulate, and the appearing thermal diffusion acceleration does not depend on the masses of the accelerated particles. As a result, the world lines of particles are curved in the same way and all processes with them become slower, which is equivalent to dilation their proper times.

Thus, the local energy deficit of the background field, giving rise to conservative thermal diffusion, reproduces the basic properties of gravity. On the hypersurface of simultaneity, where the background field is taken, effective metric, connectivity and curvature arise, and Einstein's equations for the metric follow from the energy balance in the system "source + background field".

As a result, gravity appears as a consequence of DQM, a manifestation of quantum fluctuations of particles in an inhomogeneous background field, i.e. diffusion gravity. The diffusion treatment of gravity leads to a number of observational consequences in astrophysics (dark matter effect) and cosmology (violetshift), which will be discussed in Part 6.

Thus, a fundamental consequence of conservative diffusion in DQM is the corresponding thermal diffusion in the inhomogeneous background field, where the inhomogeneity of the 
energy density of the background field is generated by the presence of matter. This thermal diffusion turns out to be the desired physical mechanism of gravity.

Since this treatment is based on quantum concepts, it implements a new form of synthesis of the theory of gravity and quantum theory. Of the two basic postulates of modern physics, about the existence of quantum fluctuations and gravity, DQM leaves only the first one as a postulate, and the second turns out to be its consequence, i.e. the theory of gravity becomes a part of the quantum theory.

The tasks for further research are mainly the origins and mechanisms of fluctuations of the background field, while the acceptance of these fluctuations as an observational fact leads to a simple and clear physical picture of both quantum and gravitational phenomena, greatly simplifying the situation in the foundations of physics.

\subsection{What is the physical meaning of Einstein's equations?}

One of the main postulates of GR is that gravity is generated by the energy-momentum of the source. In the diffusion treatment, the energy of the background field around the source is reduced to the value of the energy of quantum fluctuations of its particles (i.e., their rest energy) and the kinetic energy obtained from the background field during the formation of the source. The total energy of the source is formed from the energy to which the energy of the background field in its vicinity has decreased. Therefore, the sum of the total energy of the source and the energy deficit of the background field around it is equal to zero.

The decrease in the energy density of the background field near the source and its smooth recovery with distance from it can be described in the diffusion picture, in terms of gravitational potential or the curvature of space-time.

In the general case, the condition for the balance of the energies of the source and the background field around it should be written locally, i.e. through the energy and momentum density of the source on the one hand, and the function characterizing the space-time geometry, on the other hand, which leads to Einstein's equations for gravitational field.

A clearer physical picture also clarifies the issue of the energy of gravitational field in the rest frame of the source, and in all other reference frames this energy can be found by transforming its values in the rest frame of the source.

Thus, the theory of diffusion gravity is the next step in understanding the nature of gravity after GR. From this theory, as shown above, both the principle of equivalence and the connection of field quantities with the energy-momentum of matter naturally follow. Therefore, the new treatment naturally reproduces the formalism of GR, but not as a formal mathematical model, but as a geometric method for describing diffusion gravity, when it is not necessary to clarify its microscopic mechanism.

\section{Problems of cosmology and their solutions}

The main problems of relativistic cosmology $[5,10,18]$ at the beginning of its formation were three problems, to which, by the irony of history, researchers have so far paid almost no attention:

36. The double redshift paradox in relativistic cosmology.

37. Is it necessary to take into account aberration in cosmology?

38. Does the rate of proper times change during expansion?

The Friedmann models [10] are based on the assumption that the proper time rate is constant during the expansion. As the result, at joint taking into account the contributions of wavelength extension and the Doppler effect, required by GR, the double redshift paradox arises.

As it was shown in the papers [42], the exclusion of this assumption and the transition to a more general case, admitted by GR, to the slowing time cosmology (STC), solves this 
paradox. The new model does not contain dark energy and other problems of previous models with the early universe, i.e. a number of problems of previous models are being solved:

39. Are there cosmological constant and dark energy?

40. Problems of Friedmann models of cosmology and their solutions.

The problems solving by the new model on the basis of observations are:

41. Does dark matter exist?

42. What are the curvature and global structure of the universe?

Solutions of these problems of cosmology are presented in the papers [42] and in books [45,48].

\subsection{The double redshift paradox in relativistic cosmology}

In static space, photons from sources receding from us will come to us with Doppler redshift. Moreover, in our rest frame, this displacement is already present at the beginning and does not change further, i.e. photons will arrive at us with the initial Doppler shift regardless of the distance [22].

If space expands, but the source rests relative to us (non-comoving the expansion), then photons from such a static source are emitted without Doppler shift. Along the path, the wavelengths of these photons will increase due to the expansion of space and the photons will arrive at with expansion redshift. This purely cosmological redshift increases with distance.

These are two independent mechanisms of the cosmological redshift: the first, the Doppler effect for sources receding from us, exists already at the beginning, and the second, the stretching of the wavelengths of photons from any sources, arises during propagation.

In expanding space, objects comoving the expansion recede with growing by distance velocities. Therefore, in our rest frame, photons from such sources are emitted with the Doppler redshift, and then acquire an additional redshift along the path due to the stretching of their wavelengths. This can be easily verified by introducing another source near such a source, which is at rest in our rest frame, the photons from which will come without the Doppler effect and only with stretching of wavelengths.

Thus, the cosmological redshift of the comoving the expansion objects contains the contribution of both mechanisms and not one of these effects alone. But under the assumption that the initial wavelength coincided with the wavelength of our photons here, the really observed redshifts correspond to the contribution of only one of these mechanisms. So, the theory predicts two types of redshift, while observations show the presence of only one of them. This is the double redshift paradox.

At low velocities, the contribution of both types of redshift is the same, which was the reason for the confusion in the treatment of the nature of the redshift. If the velocities are not small, then the Doppler effect must be in a relativistic form and dissimilarities arise.

In the standard formulation of relativistic cosmology, only one of these two mechanisms was indicated as the cause of the redshift. Some authors pointed out the Doppler effect as the cause, while others - stretching the wavelength along the way due to expansion of space. This duality in explaining the redshift was a problem, but instead of solving it, a myth was created that these two treatments are equivalent ways of describing the same physical phenomenon. Based on this myth, when taking into account one of the effects, the other was completely ignored. By this, the theory was in fact fitted to observations, since accounting both types of redshift would lead to a doubling of predictions, in complete contradiction with observations.

In the papers [42] it was shown that the agreement of theory with observations is achieved not by ignoring one of the two independent physical phenomena, which is erroneous in GR and led the development of the theory to a dead end, but by searching for a third mechanism admissible in GR that compensates the contribution of one of the two types of redshift. 
The key to solving the double redshift paradox was given by the rethinking of the assumption that was considered obvious, that the initial wavelength of the photon is the same as the wavelength of our photons here. In GR, this is not the case, and therefore returning to a more general case allowed one to solve the double redshift paradox (see Section 6.3).

\subsection{Is it necessary to take into account aberration in cosmology?}

Aberration, a change in direction to a light source and its apparent luminosity due to relative velocity, is one of the main optical effects in astronomy, allowing to eliminate the optical distortions.

However, in relativistic cosmology, where the receding velocities of sources are rather high, aberration has not been taken into account (it was taken into account only in models without space expansion). The reason for this was the interpretation of redshifts as a result of stretching of wavelengths.

As shown in Section 6.1, both stretching and the Doppler effect contribute to the cosmological redshift. But in relativistic kinematics, if the source is receding, then the Doppler effect and aberration are determined by the same factors, and therefore the presence of the Doppler effect leads to aberration also. These two effects affect the properties of the stream of photons together - the first reduces the frequency of photons, and the second - the number of photons arriving at a given solid angle.

At an increase in the solid angle of the cone, the number of photons in the cone of the former solid angle will decrease, which leads to a decrease in apparent luminosity. This decrease in the density of the light flux coming from receding objects must be taken into account in the expanding universe. In this case, the aberration leads to the fact that receding sources will appear dimmer than those which was rested relative us at the same distance.

Unreasonable ignoring the aberration, as has been done so far, led either to a systematic overestimation of the distances to sources, or to a systematic underestimation of their absolute luminosities. To explain such a "mysterious" dimming, various hypotheses were then introduced, the main of which was the hypothesis of accelerated expansion, to justification of which the dark energy hypothesis has been introduced.

\subsection{Does the rate of proper times change during expansion?}

The double redshift paradox in the expanding universe has no solution in Friedmann's models, where the rate of proper times is constant. This fact makes these models inconsistent due to the contradiction of their real prediction (double redshift) with observational data (single redshift).

The first step to solving the paradox is to find an answer to the question: what was the initial wavelength of photons from distant sources?

The cosmological principle requires that the average density of matter everywhere be the same, but only under the condition that these densities are taken everywhere at the same moment of local proper times, i.e. on the "hypersurface of the same age". Therefore, from GR and the cosmological principle it follows that the wavelength of a photon, when it is emitted in an earlier epoch, coincides with the wavelength of the same photon in our place only in the same early epoch (without taking into account the Doppler effect). The wavelength of a photon emitted far from us in the epoch of 10 billion years after the Big Bang in terms of the proper time there should coincide with the wavelength of such a photon here also in the epoch of 10 billion years after the Big Bang in terms of proper time here. This means that for the wavelengths of photons in different places, emitted at different epochs, the cosmological principle does not impose restrictions.

Therefore, in order to take the second step towards solving the double redshift paradox, it is necessary to answer the question of how are the wavelengths of photons emitted in the same place, but at different epochs related to each other? In particular, did the wavelength of 
the photon emitted here in our place in an earlier epoch differ from the wavelength of such a photon now?

In GR, there is freedom in choosing the rate of the proper times in different epochs of cosmological time and the choice can be made only on the basis of observational data. These data show that the double redshift paradox is solved only if the rate of proper time everywhere slowed down during the expansion.

At variable proper time, it is necessary to choose the time of one of the epochs as a standard. It is natural for us to choose the rate of our time as a standard, since we compare signals from previous epochs with signals in our time. Such a model of slowing time cosmology (STC) does not contain the double redshift paradox.

Previously, the influence on the cosmological evolution of an increase in the distances between objects was mainly studied, and STC also studies the effect of an increase in the time interval between events. At the same time, it is shown that GR is consistent with observations only at a faster rate of time in previous epochs and its dilation during expansion.

The fact is that in this case, in addition to two mechanisms of cosmological redshift, the Doppler effect and stretching, a third mechanism appears - the violetshift for photons emitted in earlier epochs. This new kind of redshift is compensated for along the way by stretching redshift and the observed redshift will contain only redshift due to the Doppler effect alone.

Observations already in the linear part of the dependence of redshifts on distance reject the models with the Friedmann metric, leading to a double redshift and agree only with the STC with a single final shift. As it was shown in [42], the new "distance modulus-redshift" relation is consistent with observational data, and describes them without dark energy or similar speculative hypotheses.

STC also leads to a number of non-trivial consequences for the early universe and allows to naturally solve the cosmological problems of previous models.

\subsection{Are there cosmological constant and dark energy?}

The explanation of the observed cosmological redshifts by the relativistic Doppler effect changes the dependence of the apparent luminosities on the magnitude of the redshifts, and also leads to the need to take into account the aberration, which naturally explains the additional dimming of the sources

Ignoring these two circumstances, which has been practiced so far, has led to overestimation of distances or underestimation of absolute luminosities. To explain all this, an accelerated expansion was assumed, and then, to explain this, a dark energy was introduced.

As a result, the former SM of cosmology was based on the introduction of the cosmological constant, the dark energy density, the presence of which leads to repulsion between the masses, i.e. antigravity. However, the value of the cosmological constant turned out to be so small that it could not be explained not only by the zero-point vacuum energy in SM of particle physics, but also by its hypothetical generalizations. This is the cosmological constant problem, which turned out to be practically insoluble in the former SM of particle physics and cosmology.

In fact, as discussed above in the Part 2 of the paper, particle physics does not provide any, either theoretical or observational, grounds for introducing the zero-point vacuum energy. This means that cosmology can be in agreement with particle physics only in the absence of the cosmological constant.

In STC, the cosmological constant, and hence the dark energy, is absent, and Einstein's equations with the energy density of matter are quite sufficient to describe the observations. This realizes agreement between cosmology and particle physics in the vacuum energy problem.

At the same time, the background field of DQM has the energy density, the decrease of which is manifested as gravity. Therefore, in the STC, a certain analogue of dark energy takes place, but the curvature is not created by the entire energy density of this background field, but 
only by that small part of it that has passed into the energy of matter, and the density itself decreases with time. Therefore, there is no problem of the cosmological constant in the STC.

\subsection{Problems of Friedmann models of cosmology and their solutions}

In the Friedmann models, a number of cosmological problems arose, in particular, the problems of horizon, homogeneity, flatness, entropy and initial singularity. There are no such problems in the STC and the main reasons are changes in the evolution equation and the fact that in the early epochs the light velocity was much high and the radius of the horizon increased at a high velocity than the current light velocity.

Flatness and fine tuning problem. In the Friedmann model, in the early epochs, space becomes flat with high precision. There is no flatness problem in the STC, since the curvature parameter in the early epochs, as it turned out, decreases. Therefore, there is no need for the socalled "fine-tuning", which caused problems in the previous models.

Homogeneity, isotropy and horizon problems. In Friedmann's model, these problems arose due to the fact that as the universe expanded, the size of the causal region (horizon) grew more slowly than the size of the universe itself. The CMB has ceased to interact with matter after the epoch of recombination and the radiation densities in the causally unrelated regions could not be leveled in any way, but from these regions, on average, isotropic and homogeneous flows come, which was inexplicable. In the STC, these problems do not exist, since the largescale homogeneity and isotropy of the distribution of matter were the consequences of the causal relationship of different regions under conditions of a faster light velocity and faster evolution.

In the epoch of dominance of radiation and in the recombination epoch, particles of matter diffused as a small impurity in the high-temperature gas of photons. In those epochs, almost ultrarelativistic particles diffused rapidly, leveling out any noticeable irregularities in the distribution of matter and radiation in causally related regions. Since in the STC the light velocity was faster in terms of the time of our epoch, and the radius of curvature of the universe and the size of the causal region also increased faster in the early epochs than in the Friedmann model, the problem of the horizon does not arise in the STC.

\subsection{Does dark matter exist?}

Cosmological models introduce limits for the density of non-baryonic dark matter. There is no such need in the STC, although in principle an insignificant admixture of such matter is not excluded. In any case, both the practical absence and the presence of dark matter in small volumes only slightly changes other parameters of the theory, and therefore there is no problem of dark matter in the STC.

A preliminary study of the possible stretching of the Newtonian potential from the point of view of a diffusion treatment of gravity in early epochs showed that the observed effects, usually attributed to dark matter, can be explained by such stretching [42].

Conservation of the energy of the background field in the expanding volume leads to a new scenario in which the values of the potential are preserved at the boundaries of this volume, which means that in the physical volume they are stretched. As a result, in the expanding volume, the values of gravitational potential will comoving the expanding layers.

The radius of gravitationally bound region grows over time due to a decrease in the expansion rate, and different layers around the galaxy stop expanding at different times. Thus, as further the layer was located from the galaxy, as longer it expanded and thickened, while the potential difference at its boundaries remained unchanged. This shifts the values of potential around the galaxy proportionally to the distance and, as a result, gravitational acceleration decreases in inverse proportion to the distance, as in centrifugal acceleration.

This fact naturally explains well-known empirical facts such as plateaus in rotation curves and velocity-mass relationships for galaxies and velocity dispersions in galaxy clusters. 


\subsection{What are the curvature and global structure of the universe?}

The infinity of space in Newtonian cosmology led to a number of problems that were practically insoluble. In relativistic cosmology, there are also models of an "open" universe of infinite volume (with flat and hyperbolic spaces), in which the same problems arise.

At first, the possibility of a "closed" universe of finite volume in the form of a threedimensional sphere seemed to be one of the main advantages of relativistic cosmology. Moreover, as it turned out later, it is more natural to explain the Big Bang in a space of a finite volume than in an infinite space. However, in the Friedmann model, it was not possible to match the parameters of the closed model with observational data, and such models were practically out of sight of researchers.

But in STC, the curvature of space in our epoch does not play such a decisive role as in Friedmann's models. In this case, the model of a closed universe turned out to be quite admissible for those parameters that describe the observational data. Therefore, the possibility in the new paradigm to restore the models of the finite volume universe may become one of the most radical changes in the physical picture of the world.

\section{From SR and GR to local and global theories of relativity}

Both in SR and in GR, local and global relativistic effects were described without emphasis on the fundamental differences between them. In the paper [44] it is shown that each of these two classes of effects is universal and therefore is actually described by a separate theory.

First, descriptions in local frames of reference (LFRs) related by the local Lorentz transformations form the local relativity (LR). The principle of locality makes it possible to apply LR to non-inertial LFRs, and the principle of equivalence to LFR in a gravitational field:

\section{LR is a local form of $S R$ and for this reason it is universal.}

Secondly, the description of phenomena in extended regions using global FRs, built from LFRs on hypersurfaces of simultaneity, also turned out to be universal, applicable in both flat and curved space-time, and therefore forms a separate theory, the global relativity (GIR):

\section{GlR as a physical part of GR on hypersurfaces of simultaneity.}

As a result, the local parts of SR and GR constituted the LR, and their global parts in terms of physical coordinates formed the GIR. Thus, fragments of SR and GR turned out to be parts of two universal theories, each of which describes in a unified way the phenomena both in flat and in curved space-time.

The clarification of this circumstance made it possible to describe the phenomena of gravitational physics on a purely physical basis, using the methods of these theories, which removed a number of problems that, within the framework of GR, did not have an unambiguous solution for a long time:

\section{Consequences of LR and GlR for quantum and gravitational physics.}

Classical mechanics at one time was reformulated without changing its physical foundations - from the form of Newtonian mechanics it took more universal forms of Lagrangian and Hamitonian mechanics, which mutually complemented each other. LR and GlR, being formed from the physical parts of SR and GR, also proceed from their physical foundations, but, describing different aspects of physical phenomena, they complement each other.

LR and GIR are described in more details in the paper [44] and in the book [48]. 


\subsection{LR is a local form of SR and for this reason it is universal.}

In the vicinity of each point in space, it is possible to enter LFRs, the speeds and accelerations of which may differ in magnitude and direction. Each of them consists of a basis in the form of a test particle of small mass, to which a non-rotating local tetrad is attached. A tetrad, four mutually orthogonal unit vectors in space-time, from a physical point of view, represents three standard scales that form a spatial triad, as well as a standard clock. An event, a physical phenomenon at a given point in space at a given moment in time, is a world point in a 4-dimensional space of events, and a continuous chain of events is a world line.

Classical mechanics is based on the principle of relativity, according to which all LFRs in a given place are equal in terms of formulating physical laws. The coordinates and time of the event in the two LFR are linked by Galileo's transformations. The time evolution of particles and fields is given by the equations of motion following from the principle of least action.

$\mathrm{LR}$, which is a local form of SR, consists in supplementing classical mechanics with the principle of the constancy of the speed of light in all LFRs [3]. This leads to the presence of invariant (light-like) isotropic lines in the event space. As a result, the time axis acts not just as the fourth coordinate, but becomes the coordinate that makes the event space a 4-dimensional pseudo-Euclidean space. The transition from one LFR to another, moving relative to the first, then occurs in the form of a rotation of the spatial and temporal coordinate axes towards each other. As a result, the simultaneity of events turns out to be relative and different LFRs have different hypersurfaces of simultaneity. In this case, the transitions between the LRO are given by the local Lorentz transformations.

Time intervals are measured using single events. Distance measurement, i.e. comparing them with the standard of length is possible only by simultaneously fixing the ends of the scales, i.e. using simultaneous events. The relativity of simultaneity, i.e. the difference in the hypersurface of simultaneity, leads to basic relativistic effects - slowing down of proper times and contraction of scales.

The first and most frequently used application of LR is the case of a flat space without gravity, when LFRs become local inertial FRs and are part of the global inertial FR. All the concepts and relationships of LR given above were discovered in this particular case, within the framework of SR, which is the theory of phenomena in global inertial FR. Local inertial FRs in different places of the given inertial FR are mutually resting and therefore for the locality of LFR it is enough to be limited to a small area of space, and the time limit is not necessary.

But in flat space-time without gravity, there are non-inertial LFRs, which are also described by LR. This follows from the fact that acceleration a does not locally affect the rate of time and the lengths of the scales (the property of locality). For weak accelerations, the terms of order $\mathbf{a}^{2}$ can be neglected and the locality property takes place, because changes in the rate of time and the length of the scales can depend only on an even degree of acceleration $\mathbf{a}^{2}$ and higher, which can be neglected due to their smallness. Under strong accelerations, there are no such direct arguments for the locality property, but experiments do not yet contradict it, and therefore, having in the form of reliance on experiments, it is often called the principle of locality. An indirect argument is that gravitational field has its analogue - the principle of equivalence, which is confirmed by experiments [4]. Considering these three circumstances, we will consider the principle of locality to be valid in all non-inertial LFR.

Hence it follows that two non-inertial LFRs in the vicinity of one and the same event are connected by local Lorentz transformations and local Lorentz invariance takes place in them. To further simplify the situation, comoving local inertial FRs are often introduced, for which the local Lorentz invariance is obvious. Moreover, for local inertial RFs, locality also means in time, i.e. during the time of comoving.

In gravitational field in resting LFR, instead of the principle of locality, the principle of equivalence takes place, according to which LR is locally valid and for LFR there is local Lorentz invariance. In the non-inertial LFRs in gravitational field, according to the principle of 
equivalence, in combination with the principle of locality, local Lorentz invariance also takes place.

Thus, LR is a localized form of SR, which, unlike SR as a whole, is applicable both in unaccelerated and accelerated LFRs, both in flat and curved space-time. In LR, the basic relativistic effects for moving local inertial FRs, time dilation and length contraction, are the results of the relativity of simultaneity. Due to the difference of the hypersurfaces of simultaneity, all phenomena in moving local inertial FRs have to be projected onto the hypersurface of simultaneity of the resting (base) local inertial FR, which leads to relativistic effects.

\subsection{GIR as a physical part of GR on hypersurfaces of simultaneity.}

In the LR, in the vicinity of each point in space, families of LFRs are introduced and local events are described in terms of local spatial coordinates and local proper times of one of the LFRs at a given point. This means that within the framework of the LR, at point A, the "Atime" of one of the LFRs is defined, and at point $\mathrm{B}$, which is further from the close vicinity of A, the "B-time" of another LFR is defined, but the common "time" for A and B is not defined [3]. The same is the case with the definition of the lengths of the standard scales.

Therefore, let us consider the definitions of times and lengths common for two LFRs in A and B. For this, at one point, chosen as the origin of coordinates, at the initial time, i.e. in the event $O$, we will select one of LFR at this point as the base one, and then for each family of LFRs at other points we will select one LFR at a time that is simultaneous with the event from the point of view of the observer in the basic LFR. At other points in time for the basic LFR, we will repeat the same procedure. If then at each moment of time in such a set of simultaneously coexisting LFRs at different points to synchronize adjacent standard clocks and continuously dock adjacent spatial triads consisting of standard scales, then we will thereby form the global FR (GFR). Even if the synchronization breaks down after a short time interval, at least for a short period of time we will be dealing with the GFR.

In this case, the physical coordinate system in space is formed depending on the symmetry properties of the physical system that must be described using this GFR. Space without taking into account the influence of fields and particles is homogeneous and isotropic, and the Cartesian coordinate system fully expresses these symmetries of space, but if the physical system has, for example, spherical symmetry, then a spherical system is constructed.

By introducing the GFR, we thereby go beyond the LR and the theory describing events by introducing the GFR, hereinafter we will call the GIR. Within the framework of the GIR in each of the GFR, physical reality appears as a set of particles and fields that instantly coexist in space on a certain hypersurface of simultaneity, where the LFRs forming the GFR coexist simultaneously in terms of the time of the basic LFR.

\subsubsection{Global inertial FR in flat space-time.}

In flat space-time, the principle of relativity and the principle of constancy of the speed of light are valid in the GIR in a global form, i.e. the laws of physics, including the constancy of the speed of light, are the same in all global inertial FRs. Thus, the GIR in this simplest case reproduces the SR.

The relativity principle in this case takes the form of the global Lorentz invariance [3]. Taking into account the homogeneity of space and time, leading to translational invariance, the Poincaré group becomes the symmetry group.

In LR, slowing down of times and contracting of lengths in moving local inertial FRs were the result of projection onto the hypersurface of simultaneity of a resting local inertial FR. In the global inertial FR, the situation is the same, but now globally.

For us, the fact is important that the above-considered global aspect of SR - the construction of a global inertial FR on the hypersurface of simultaneity of the basic LFR, turns out to be a more universal construction than SR itself. Being applicable both for non-inertial 
GFR and for GFR in a gravitational field, this construction underlies the GIR, the theory which also is universal as SR. Below the main statements and some consequences of this treatment will be briefly discussed.

\subsubsection{Extended non-inertial FRs in flat space-time.}

In a flat space-time, a set of LFRs with different speeds and/or accelerations forms an inhomogeneous extended FR. In such an extended FR, the principle of local Lorentz invariance is valid and the elements of extended FRs are only LFRs associated with other LFRs by local Lorentz transformations and shifts. In addition, relativistic causality requires that any extended FR, as a unified system of material objects, should consist only of simultaneously coexisting LFRs. Therefore, an extended FR should be built on the hypersurface of simultaneity of one of the global inertial FR, chosen as the basic one.

If one of the two clocks experiences acceleration, then the accelerated clock will dilate, since the space-time interval along the indirect world line is less than that of the straight world line. In particular, during oscillations and fluctuations of particles, their proper times are delated irreversibly in comparison with the proper time of a particle at rest in the LFR, and the processes in them occur more slowly than in an unaccelerated particle.

In a flat space-time, the construction of inhomogeneous extended FRs from LFRs on the hypersurface of simultaneity of the basic inertial FR, thus, constitutes the first stage of globalization of LR. This makes it possible to correctly go beyond the inertial FR when describing physical phenomena and will constitute the initial stage of constructing the GIR as the globalization of the LR or the separation of the physical part of GR. Further, this procedure can be used to describe events in gravitational field.

Let us note here the key role of the requirements of causality in the construction of an inhomogeneous FR, which means that the positions of parts of such an extended structure as a global FR must be simultaneously fixed in at least one global inertial FR, which will be considered a laboratory inertial FR. An inhomogeneous global FR is built precisely on its hypersurface of simultaneity, and at the same time, the spatial axes of all LFRs are projected onto this hypersurface. In particular, for a spherically symmetric physical system, the distinguished global inertial FR is the one where its center of symmetry is at rest, since only in it the system is spherically symmetric, and in all others it is ellipsoidal.

\subsubsection{Gravitational field in a resting global FR.}

Two facts were basic in understanding the physical foundations of the GIR for noninertial FRs in flat space-time The first fact is that a global FR is a system of physical coordinates as a set of standard scales, equipped with a standard clock, connected together at a given time of the basic global inertial FR. This means that the global FR should be a set of bodies coexisting simultaneously in at least one global inertial FR, and therefore should be built on the hypersurface of the simultaneity of this inertial FR. The second fact is the validity in these global FR of the principle of locality, expressed by local Lorentz invariance. This significantly limits the general covariance on which GR was based, since non-Lorentz transformations are excluded.

The main task facing the GIR is to build a theory of gravitational fields of real sources. But, to solve this problem, there is no need for moving FRs, since the effects of gravity in their pure form are manifested in a global FR resting under the field's source. In this case, the principle of equivalence states that LFRs at rest in this gravitational field are equivalent to accelerated LFRs in flat space-time. This means that in gravitational field, as in the accelerated LFR, the LR is locally valid and therefore the neighboring LFRs resting in gravitational field on the hypersurface of simultaneity of the basic LFR are related by the local Lorentz transformations.

Initially, the general covariance was considered as a heuristic principle, from the general consequences of which the physically realizable part should be selected using the local Lorentz invariance [4]. In all tests of GR in experiments, it was implicitly taken into account the fact 
that the physical content of GR is given only by this part. To reflect this circumstance in the formalism of GR, special methods were developed, such as tetrad, Hamiltonian, and bimetric formulations, which partially solved this problem. However, each of them did not solve the problem as a whole, and it was not possible to combine them into a single method.

In the GIR, initially based only on the physical coordinates of LFR, there is no problem of excluding non-physical aspects, but the above methods, which were developed within the framework of the formalism of GR, can be used as GIR tools. Thus, the GIR, as GR in physical coordinates specified on the hypersurface of simultaneity of the basic LRR, retains the physical part of GR, including all experimentally observed consequences, and excludes the nonphysical part of GR.

\subsection{Consequences of LR and GIR for quantum and gravitational physics}

At extrapolating GR in an area where there were no direct checks, the picture of physical reality was often distorted. The theory, being excessively general, also included non-physical elements, such as arbitrary reference frames and their coordinates, so at applying GR to real systems with physical coordinates, it was always necessary to clarify which part of it describes real events and which does not.

In simple cases this is not difficult, but under extreme conditions the basic concepts of the theory had to be brought into correspondence with relativistic constraints in the vicinity of each local system. Therefore, the task of separating the physical part of GR from the nonphysical was a fundamental problem, the solution of which was to turn the realistic part of GR into a consistent physical theory, all the consequences of which could be fully trusted.

GIR, as such a physical part of GR, operates with physical coordinates based on the determination of distances and times together with the physical components of the metric, expressing the geometry of space-time in a given gravitational field. The transition from the product of some physical coordinates, measured by standard scales and clocks, to other physical coordinates is done for simplification, taking into account symmetries, as in the transition from Cartesian coordinates to spherical ones. However, such a transition cannot change the spacetime geometry in the rest frame of the source and only introduces geometric and kinematic distortions into this picture.

In particular, LR and GIR describe local and global properties of gravity, separating field effects from the effects of motion. Such separation can be performed by different methods, in particular, using the bimetric formalism, where one metric describes the geometry of the global FR, and the other describes the geometry of space-time, and the energy and angular momentum of gravitational field are described by tensors.

In the GIR, the evolution of extended objects, such as stars, fields and, ultimately, the universe as a whole, is described in terms of two types of time, each of which expresses only one of the two basic properties of physical time. The proper time $\tau$ expresses the local rate of physical processes in the LFR, description of an extended object as a collection of simultaneously existing particles is made in terms of world time $t$, which expresses the global simultaneity of events in the global FR.

In particle physics, the GIR leads to gravitational regularization of loop diagrams at the Planck length (see Part 3), in relativistic astrophysics of compact objects it leads to the theory of frozars (see Part 4), and in cosmology - to a model of slowing time cosmology on hypersurfaces of simultaneity, where the stretching effects are compensated by the initial violet shift and only the contributions of the Doppler effect and aberration are observed (see Part 6). 


\section{The contours and perspectives of new physics}

In Part 1 of the paper, it was noted that the crisis occurred in fundamental physics was caused by a departure from the methodology of natural sciences, and a return to the latter, taking into account some new ideas, could lead to new physics. In Parts 2-7, it was shown that this is indeed the case, and below we will briefly consider some of the results that allow us to outline the contours of the new physics and its prospects.

46. The contours of new physics.

47. Scientific perspectives of new physics.

48. Social perspectives of new physics.

The perspectives and methodology of physics are considered in more detail in the book [49].

\subsection{The contours of new physics.}

In quantum mechanics, particles fluctuate, and therefore the preservation of the concept of Euclidean space and a classical isolated particle together with the conservation of energy and momentum of such a particle is quite possible if one considers these fluctuations as evidence of the existence of the fluctuating background field. According to the principle of relativity, this field should be scalar, and random interactions of particles with it should lead to conservative diffusion of the ensemble of these particles. The velocity of particles between interactions with the background field must also be invariant and therefore equal to the speed of light, and the thermal energy of fluctuations manifests itself as the rest energy of particles. It was possible to formulate such a more general theory of quantum phenomena, diffusion quantum mechanics, DQM, based on physical principles. In a uniform background field DQM reproduces the formalism of quantum mechanics, including quantum statistics, by using the classical concepts.

Taking into account the reverse effect of a large concentration of particles on the energy density of the background field, it turns out, reproduces the properties of gravity. The diffusion treatment of quantum theory therefore leads to a diffusion treatment of gravity as a deficit in the energy density of the background field in the vicinity of massive bodies. In this case, this deficit arises due to the fact that the rest energy of a massive body is that thermal energy, which is spent by the background field on fluctuations of the particles of this body. The energy balance between such a body and the background field in its vicinity leads to Einstein's equations and all the corresponding physical consequences of GR.

If the universe has such a background scalar field, then during the expansion of space, its energy density should decrease with time everywhere. Since this should lead to a decrease in the intensity of quantum fluctuations of particles, including their rest energy, therefore, proper times should slow down during the cosmological expansion. Then, in the past, time passed faster, which means there was an initial violet shift of the frequencies of photons emitted in earlier epochs. But this violet shift must be compensated for by the redshift in the way and the photons should have come to us only with redshift due to the Doppler effect associated with the receding speed of sources. In Part 7, it was noted that the luminosity-redshift formula of the slowing time cosmology (CST) is consistent with the redshift data of distant objects.

In addition to this, the energy density of the background field around the centers of galaxies decreases irregularly during the expansion, since it was initially irregular, and the expansion itself was slowing down. It turned out that taking into account the influence of the initial inhomogeneity and the rate of deceleration on the final distribution of the energy density of the background field, and hence gravitational potential, leads to a modification of the Newtonian potential in approximately the same way as it is required to describe the rotation curves of galaxies and velocity dispersions in their clusters.

Relativistic fields in the covariant formulation of QFT initially have states with both energy signs. In the SF treatment, particles with negative energy go only backward in time and describe antiparticles with positive energy going only forward in time. This treatment is quite consistent in the sense that at an accurate definition of the time integrals in the expression for 
the action function, it leads to positive probability states only. In this case, there are Lagrangians that do not lead to zero-point vacuum energy and therefore the vacuum energy of quantized fields and strings is equal to zero. As a result, string theories become trivial, since they will not have a conformal anomaly, and therefore there will be no restrictions on the dimension of space.

In QFT, the external gravitational field of high-energy quanta in loop diagrams slows down their proper times relative to the time of distant observers. Moreover, at the Planck length, gravitational radius of Planck energy quanta, all processes freeze for these observers and therefore do not contribute to the probabilities of processes. Freezing of quantum fluctuations means a strong redshift of high frequencies up to zero, i.e. gravity of quanta leads to their selfregularization. The nonlinearity of the fields increases gravitational effects, and hence freezing too, which further suppresses the contributions of high energies. The finiteness of the corrections from small distances makes the nonrenormalizable models consistent if these corrections are small up to the Planck energy, which is the case for quantum gravity. For gauge fields and quantum gravity, the invariant Planck cutoff of energy integrals gives upper limits for loop corrections, which turn out to be small and the perturbation series converge.

Thus, QFT, including quantum gravity, is finite at quantization in the SF treatment taking into account gravitational self-regularization. This means that QFT was initially correct and consistent, which explains its extraordinary success in particle physics, and these corrections to the historically formed elements of its formulation finally clarified these circumstances.

Gravitational freezing of proper times w.r.t. world time on the hypersurfaces of simultaneity leads to the fact that collapsing objects freeze when their surface approaches their gravitational radii, all the time remaining outside this radius. A thin dust shell freezes outside its gravitational radius, while its interior remains flat. When a star collapses, the positions of its particles, as in any extended object, must be specified on the hypersurfaces of simultaneity, marked by the moments of world time. In this case, the surface of a dust star then freezes over its gravitational radius, and this asymptotic behavior of the world lines of the star's particles on the surface is invariant. The center of the star freezes before other layers, after which the entire structure of the star quickly freezes. This means that a specifically GR phenomenon gravitational time dilation - is the physical mechanism that stops the collapse in terms of ordinary astronomical time around the star.

All this can be seen by the example of the exact solution of GR equations for a homogeneous dust star in terms of world time, found by Oppenheimer and Snyder. The plots of the world lines of the layers show that the dust star, at collapse, goes into the state of frozar, an object with a completely frozen structure. The inner layers remain locally homogeneous and freeze near their effective gravitational radii formed by the matter inside each layer, as well as the influence of the outer layers on the course of their proper time. Before freezing, the density of sufficiently massive stars is lower than that of a neutron star, and therefore, if their cores did not explode before, then the collapse of such stars occurs as in a dust star with the formation of a frozar. The rotation of the stars freezes even before the surface reaches the external boundary of the ergosphere, so that the rotating frozar has no horizon and no ergosphere. Accretion on frozar leads to the freezing of the falling matter above the surface with the formation of a heterogeneous landscape of flattened mascons. Frozars do not merge, but stick together around gravitational radius of a multifrozar system, forming, together with ordinary matter, a frozar cluster. Supermassive frozars, or superfrozars, are formed mainly as such inhomogeneous clusters. Frozars and their clusters are not "bald" and may have a "hairstyle" and asymmetric structure. The inhomogeneities of their field can be detected by gravimetry, inhomogeneities of shadows, redshifts and orbits of matter.

In the theory of relativity, processes can be described in both local and global FRs. Each of these two methods is universal and therefore actually forms a separate theory. Descriptions in LFRs connected by local Lorentzian transformations form LR, which is universal, since the principle of locality allows it to be applied to non-inertial LFRs, and the principle of equivalence - to LFRs in a gravitational field. Thus, LR is a localized form of SR, which is applicable both in non-accelerated and accelerated LFRs, and in both flat and curved space-time. 
GFR is a set of simultaneously coexisting LFR and, therefore, each of them must be built on some hypersurface of simultaneity. The introduction of the GFR, thus, means going beyond LR and the theory describing events by introducing such GFR is the GIR. In GIR, physical reality appears as a set of particles and fields coexisting on the hypersurface of simultaneity of one of GFRs. The GIR operates with physical coordinates based on the determination of distances and times with physical scales and clocks, and therefore is a physical part of GR. LR and GIR describe the local and global properties of gravity, separating the effects of the field from the effects of motion by different methods previously proposed in the framework of GR.

\subsection{Scientific perspectives of new physics.}

The perspectives of the part of the new physics discussed above, like any innovation in science, have two aspects - scientific and social ones. At first, consider the scientific perspectives that are of interest for active researchers and unprejudiced readers.

The diffusion treatment of quantum theory, DQM, leads not only to a new and consistent treatment of the well-known phenomena of quantum physics, but also opens new perspectives for the development of particle physics, especially in the direction of unifying of interactions and composite models of particles.

The connection between the fluctuating background field of the DQM and the condensate of the scalar field, which in SM generates masses (and hence rest energy) of the particles, requires a more detailed study.

Also intriguing is the question of the existence of an extra dimension of space, which can also bring greater clarity to the sources of fluctuations of this background field or fluctuating condensate. This is especially interesting in the conditions of the cosmological expansion of our space and clarification of the questions about where those more and more new areas of space appear from, which were not in our world in the past, and how they are filled with condensate.

The diffusion treatment of gravity, a new and unexpected synthesis of quantum and gravitational physics, opens up completely new prospects for further research both in the theory of gravity itself and in its applications in astrophysics and cosmology. The fact that this treatment gives a natural mechanism for the appearance of the dark matter effect, associated with the comoving to expansion of gravitational potential, shows the possibility of surprises at studying gravity in expanding space, especially in scenarios of the formation of galaxies, clusters and the cosmic web.

In slowing time cosmology, after specifying the details of expansion models, it becomes possible to establish, without artificial hypotheses, the real distances up to objects and their true luminosities, to determine the most realistic scenarios for the expansion and the course of cosmic evolution from early periods.

Quantization in the framework of SF treatment and gravitational regularization make it possible to formulate and solve in a new way the problems concerning composite models, models of unification, field condensates and contributions beyond the perturbation theory.

The frozar theory, as a consistent theory of relativistic collapse, can predict new phenomena in the astrophysics of compact objects, especially in the case of supermassive frozars, the structure of which is the most inhomogeneous. In the earlier universe, there may also be unexpected scenarios for the development of rather dense regions, gravitational collapse of which must be taken into account together with the dark matter effect, when the expansion deepens gravitational potential.

In the new physics, SR and GRT take new forms - LR and GIR, which, being free from internal problems, can be applied to all areas of physics already as purely physical theories that clearly define the physical aspects of phenomena in each of their areas of application. Under these conditions, a number of changes are to be made in these areas, in particular, in QFT in accelerated FRs and in an external gravitational field, where the GIR will make it possible to clearly separate real and fictitious quanta. 
Notice, that the predictions of further directions of research will now be more definite and scientifically substantiated than it was until now. This is due to the circumstances that they will be based not on hypotheses, but on general physical theories proceeding from general physical principles, taking into account the most probable directions of their further generalization, arising from their internal logic and a number of still unexplained facts.

\subsection{Social perspectives of new physics.}

The social aspects of the perspectives of new physics include the perspectives for its recognition and further development by the scientific community, which is important not only for researchers, but also for teachers, science administrators, as well as popularizers and the general public.

At the same time, the recognition of not particular and insufficient, but cardinal changes in the paradigm is very painful for most part of the scientific community and therefore it can last for a long time. The fact is that the modernizations described in the paper concern the foundations and the most important applications of basic theories, as well as the contradictions of the methodology of natural sciences the main hypotheses of the crisis period, which ends this crisis and opens the way to new physics. The rapid recognition and further development of these innovations by the world scientific community are also hindered by a number of seemingly unusual circumstances.

First, it is an attitude of distrust based on the presumption of erroneousness, when such changes are proposed by a lone researcher, moreover little-known, and even from a country little-known in terms of fundamental discoveries in physics. However, theoretical physics differs from experimental physics in that most often new cardinal ideas were put forward and on their basis new basic theories were built not by large groups, but by individual researchers, as a rule, who were not part of large groups and did not share their prejudices. In this case, in the course of solving the above problems, the author had information about the successes and mistakes of all such groups, but was as far away and isolated from them as possible, while retaining the main value of the researcher - sovereignty.

The second is the understanding by many of the fact that if all this is admitted, then most of the works of many leaders of the current scientific community and a huge number of their followers will lose their value, and their authority based on the creation and promotion of hypotheses which at the end appeared inconsistent. The third circumstance is connected with the same fears - this is the legacy of the crisis in the form of a general fear of taking responsibility for approving a logical and reasonable new scientific theory if it significantly changes the current paradigm. Such infantilism and conformism occurs in communities, where a number of generations were required to agree in basic doctrines and loyalty to leading groups as a condition for a comfortable life and advancement in the community.

Before this crisis, the leaders of the community of physicists, or "true authorities", on whose opinion they were oriented, were only those researchers, at least one of whose ideas was confirmed experimentally. Making a career in science based only on hypotheses was risky, since hypotheses, being "scientific start-ups," most often burst like soap bubbles and the careers built on them collapsed with them. But during the last crisis in fundamental physics, none of the new hypotheses was confirmed, and therefore "true authorities" ceased to arise.

Under these conditions, each researcher faced a moral and ethical dilemma: a) either to make a scientific career on considered promising hypotheses, understanding the risk of its collapse in the future, b) or refrain from making a career on hypotheses as potential mistakes and try to make a real contribution to science. Solution (b) in a crisis meant, in view of the long years of searching without demonstrating "success," the displacement of such a researcher to the level of a marginal in the community of more "successful" colleagues who made a more pragmatic decision (a).

During the crisis, pragmatic members of the scientific community, in the absence of real discoveries, successfully advanced in their careers by constructing and comprehensively 
exploring a large number of unreal worlds and an unbounded number of their important details, and having united and occupying key positions in the infrastructure of science, they turned out to be unsinkable. This made their choice a win-win and even more attracted new generations to them, for whom the abandonment of the sovereignty of a researcher for the sake of a seemingly successful career had already become natural. This institutionalization of the departure from the ethics of the natural sciences led to the substitution of science by its imitation, resulting in a long crisis in fundamental physics discussed in Part 1.

The development of science usually goes through the competition of generations of researchers and what has not been done by one generation, even with the availability of opportunities, will be done by other, more rationally acting generations. But those researchers who, having abandoned the conformism and like-mindedness demanded of them at the crisis, still can to return to the objectivity and open-mindedness that lie at the basis of the methodology of natural sciences, then they still have a chance to make a real contribution to science, following Eastern wisdom "No matter how far you have gone on the wrong way, come back!".

\section{Conclusion}

The considered fundamental problems, some of which have remained unsolved since the creation of the basic theories of modern physics, as we see, have quite acceptable solutions and, at the same time, without new hypotheses. The fact that for this it turned out to be sufficient to proceed from the available facts, verified in many experiments and observations, allows us to hope that these solutions are correct or at least close to the truth. They made it possible to explain inexplicable phenomena, unify theories of different phenomena and predict new ones.

If until now quantum theory and relativity theory were obviously incomplete in their foundations, now the situation becomes sufficiently improved and simplified. Researchers who rethink these basic theories with new solutions will experience a double feeling. The first is the satisfaction that these theories have reached a certain level of completeness and even perfection. The second is the interest to the new perspectives opening by new solutions, since improved tools allow solving a wider range of problems and open ways for solving still unresolved ones.

In conclusion, notice that the main thing here is a gradual clarification of the true picture of reality through an objective and unbiased analysis of facts with real, not illusory, solving of problems. In this regard, any clarification of the foundations of the picture of physical reality, especially in a number of its aspects, is an absolute value that does not lose its significance in the future, even if it becomes a part of an even wider picture.

\section{References}

1. Dirac P.A.M. (1930) Proc. R. Soc., A 126, 360.

2. Ehrenfest P. (1927) Zs. Phys., 45, 455.

3. Einstein A. (1905) Ann. d. Phys., 17, 891.

4. Einstein A. (1916) Ann. d. Phys., 49, 769.

5. Einstein A. (1917) Sitz. Preuss. Akad. Wiss., 142.

6. Fényes I. (1952). Z. Phys., 132, 81.

7. Feynman R. P. (1948) Phys. Rev., 20(2), 367.

8. Feynman R. (1949) Phys. Rev., 76, 749.

9. Feynman R. P., Hibbs A. R. (1965) Quantum Mech. and Way Integrals. McG.-H.

10. Friedmann, A. (1922). Z. Phys., 10, 377.

11. Fürth R. (1933) Zs. Phys., 81, 143.

12. Grabert H., Hänggi P., Talkner P. (1979) Phys. Rev. A 19, 2440.

13. Guerra F., Marra R. (1983) Phys. Rev. D 28, 1916.

14. Hubble E. (1929) Proc. Nat. Acad. Sci., 15, 168.

15. Nelson E. (1966) Phys. Rev., 150, 1057; (1985) Quantum Fluctuations. Pr.U.P.

16. Kerr R. (1963) Phys. Rev. Lett., 11, 237. 
17. Klein O. (1961). Ein. Prob. allgem Rel. in W. Heisenb. Phys. unz. Z., p. 58.

18. Lemaître G. (1927) Ann. Soc. Sci. Bruxel., A47, 49.

19. Madelung E. (1926) Zs. Phys., 40, 322.

20. Marshall T. (2012) Astroph. \& Space Sci., 342:329.

21. Maxwell J. K. (1996) A Dynamical Theory of the Electromag. Field, p. 76-77, W.S.P.

22. Milne E.A. (1934) Q. J. Math. Oxf. 5, 64; Farley F.J.M. (2010) P. R. Soc. A., 466:3089.

23. Oppenheimer J., Snyder H. (1939) Phys. Rev., 56, 455.

24. Pauli W., Weisskopf, V. (1934) Helv. Phys. Acta, 7, 709.

25. Pauli W. (1940) Phys. Rev. 58, 716.

26. Pauli W. (1943) Rev. Mod. Phys. 15, 175.

27. Pavšič M. (1999) Phys. Lett. A254, 119.

28. Schrödinger E. (1930) Sitz. Preus. Akad. Wiss., 418; (1932) Ann. I.H.P. 2(4) 269.

29. Schwarzschild K. (1916) Sitz. Preus. Akad. Wiss., 189.

30. Smolin L. (2007) The Trouble with Physics. The rise of string theory, the fall of a science, and what comes next. Peng. B.

31. Stueckelberg E.C.G. (1941) Helv. Phys. Acta, 14(7) 588.

32. Tersoff J., Bayer D. (1983) Phys. Rev. Lett. 50, 553.

33. Tolman R. (1934) Proc. N. Acad. Sci. USA, 20, 169.

34. Weinberg S. (1972) Gravitation and Cosmology. Wiley.

35. Weinberg S. (1994) Dreams of a Final Theory. Vint. B.

36. Zakir Z. (2014) TPAC, 9(2) 73.

37. Zakir Z. (2016) TPAC, 11, 1.

38. Zakir Z. (2017) TPAC, 12(1) 1; 12(1) 17.

39. Zakir Z. (2018) Astroph. \& Space Sci. 363: 30.

40. Zakir Z. (2020) Quant. and Grav. Phys., 1:001-7128; 1:002-7128.

41. Zakir Z. (2020) Quant. and Grav. Phys., 1:003-7129; 1:004-7129.

42. Zakir Z. (2020) Quant. and Grav. Phys., 1:005-7130; 1:008-7160.

43. Zakir Z. (2020) Quant. and Grav. Phys., 1:006-7132; 1:007-7133.

44. Zakir Z. (2020) Quant. and Grav. Phys., 1:010-7272 (publ. 28.11.2020).

45. Zakir Z. (2020) Diffusion Quantum Mechanics and Diffusion Gravity. CTPA, T.

46. Zakir Z. (2020) Finite Quantum Field Theory, CTPA, T.

47. Zakir Z. (2020) Theory of Frozars. Relativistic Collapse of Stars. CTPA, T.

48. Zakir Z. (2021) The Theories of Local and Global Relativity. CTPA, T.

49. Zakir Z. (2021) Foundations of Physics. CTPA, T. 